\title{
Simulation of Si:P spin-based quantum computer architecture
}

\author{
Angbo Fang ${ }^{1}$, Yia-Chung Chang ${ }^{1}$, J.R. Tucker ${ }^{2}$ \\ ${ }^{1}$ Department of Physics, University of Illinois at Urbana-Champaign, \\ 1110 West Green Street, Urbana, Illinois 61801 and \\ ${ }^{2}$ Department of Electrical and Computer Engineering \\ University of Illinois at Urbana-Champaign, Urbana, Illinois 61801
}

(Dated: September 23, 2018)

\begin{abstract}
We present a systematic and realistic simulation for single and double phosphorous donors in a silicon-based quantum computer design. A two-valley equation is developed to describe the ground state of phosphorous donors in strained silicon quantum well (QW), with the central cell effect treated by a model impurity potential. The dependence of valley splitting of the donor ground state on QW width and donor position are calculated and a comparison with valley splitting of the lowest QW states is presented. The oscillation of valley splitting is observed as the QW width or donor position is varied in atomic scale. We find that the increase of quantum well confinement leads to shrinking charge distribution in all 3 dimensions. Using an unrestricted Hartree-Fock method with Generalized Valence Bond (GVB) single-particle wave functions, we are able to solve the two-electron Schödinger equation with quantum well confinement and realistic gate potentials. The lowest singlet and triplet energies and their charge distributions for a neighboring donor pair in the quantum computer(QC) architecture are obtained at different gate voltages. The effects of QW width, gate voltages, donor separation, and donor position are calculated and analyzed. The gate tunability and gate fidelity are defined and evaluated, for a typical QC design. Estimates are obtained for the duration of $\sqrt{S W A P}$ gate operation and the required accuracy in voltage control. A strong exchange oscillation is observed as both donors are shifted along [001] axis but with their separation unchanged. Applying a gate potential tends to suppress the oscillation. The exchange oscillation as a function of donor position along [100] axis is found to be completely suppressed as the donor separation is decreased. The simulation presented in this paper is of importance to the practical design of an exchange-based silicon quantum computer.
\end{abstract}




\section{INTRODUCTION}

Recent years there have been increased research activities toward the understanding, characterization, and fabrication of a scalable quantum computer (QC) [1], due to the invention of efficient quantum algorithm and quantum error correction. Among more than twenty kinds of implementation proposals, the silicon-based QC architecture has attracted much enthusiasm, ever since Kane's seminal proposal [2] in 1998. The existing microelectronics technology can provide significant insights and huge resources during the development of a silicon-based QC, giving it significant advantage over other QC candidates.

In Kane's scheme, the nuclear spins of ${ }^{31} \mathrm{P}$ dopant atoms embedded in a silicon host, were proposed as the elementary QC units, or qubits. The resonance frequency for a nuclear spin can be tuned by the hyperfine interaction between electron and nuclear spins, which is controlled via a gate electrode, the so-called A-gate, over each qubit. Thus, a globally applied a. c. magnetic field can realize arbitrary rotations on selected nuclear spins. A J-gate (combined with A-gate), placed over the middle of neighboring donors, allows a controllable qubit-qubit interaction by varying the exchange coupling between the mediating donor electrons via the change of potential barrier between neighboring qubits. Obviously, this proposal requires a delicate spin transfer between electrons and nuclei for read-in-readout of quantum data and multi-qubit operation.

There also exist variations to the original Kane theme $[3,4,5,6]$, to circumvent the difficulties or simplify the QC implementation. Vrijen et al [3] suggest utilizing the full power of band-structure engineering and epitaxial heterostructures. The spin of a bound electron in a donor atom is used as the qubit. The qubit can be selectively tuned in and out of resonance under gate-control to realize single-qubit operation, by taking advantage of g-factor variations available in SiGe heterolayers. Larger surface gate biases could be employed to couple neighboring qubits in a controllable way by displacing the bound electrons deep into Ge-rich layers. However, this scheme suffers from the complexity of g-factor engineering and possible qubit loss due to ionization when the bound electron is dragged into the high-g region.

In this paper we study another modified version of Kane-type architecture proposed in Ref. [7]. We follow the encoding scheme by DiVincenzo et. al. [8], where universal quantum computation can be realized for composite 3-spin qubits, with only nearest-neighbor 
Heisenberg exchange interaction. A logic qubit, encoded by the subspace of three neighboring phosphorous donor electron spins, is shown in Fig. 1. Logic zero is represented by spins 1 and 2 in the singlet state $\mathrm{S}$, and spin 3 up. Logic one is a linear combination of triplet states $T_{+}$and $T_{0}$ for spins 1 and 2, with spin 3 down and up, respectively, to preserve the overall spin quantum numbers. Initializing to logic zero is achieved by cooling the system in a large magnetic field to polarize spin 3, while inducing an even greater exchange coupling $J_{\text {initial }}>2 \mu_{B} B>>k_{B} T$, between spins 1 and 2 to produce the equilibrium spin singlet. Typical parameters for $10^{-6}$ initialization error at $\mathrm{T}=100 \mathrm{mK}$ are $B \sim 1 T$ and $J_{\text {initial }} \sim 200 \mu \mathrm{eV}$.

There are two kinds of top gates to control qubit operations, shown in Fig. 1, similar to the A-gates and J-gates in Kane's proposal. One-qubit operations are implemented with the nearest-neighbor exchange in four or fewer steps, eliminating the need for gated ESR rotations. The two-qubit CNOT operation can also be performed by the nearestneighbor exchange within a 1D array, in 19 steps[8]. Readout can be performed with a single electron transistor(SET) incorporated close to individual qubit, by utilizing spincharge transduction[9].

In our design (Fig. 1), phosphorous donors are placed at substitutional sites on a plane inside the $S i / S i_{1-x} G e_{x}$ quantum well(QW). We use the composition $x=0.3$ for SiGe, which yields approximately a quantum well band offset of $\Delta E_{c}=300 \mathrm{meV}$ with respect to the barriers. The strained silicon QW not only prevents ionization of the donors during gate operation, but also reduces the valley degeneracy of the donor ground state. The $S i_{3} N_{4}$ layer right below the top metallic gates, is introduced to produce the desired gate potential and exchange coupling. The planar SET shown in Fig. 1 can be patterned into the $\mathrm{P} \delta$-layer by STM along with the individual P donor qubits, in the same lithographic step.

Our device presented in Fig.1 is somehow similar to that of Friesen et. al.[4], where one uses a $\mathrm{Si} / \mathrm{SiGe}$ quantum well to confine electrons vertically and use top gates to confine electrons laterally. Electron spins in such "quantum dots" act as qubits, with top gate voltages tuning the exchange coupling between qubits. However, the use of phosphorous donor electrons in our device provides a much smaller qubit size and a more densely integrated $\mathrm{QC}$ as we scale up the number of qubits. Furthermore, as we shall see in this paper, the available strong gate-assisted exchange coupling allows a much faster gate operation than that in the Friesen et. al. proposal, which would enable faster computation and more easily 
satisfy the condition for gate operation duration v.s. qubit decoherence time required by quantum error correction principle. By using naturally bound donor electrons as qubits, we avoid additional top gates used to provide lateral confinement and the problem of precisely controlling the number of electrons in each quantum dot.

The issue of decoherence of qubits is of critical importance for the construction of a realistic QC. The quantum error correction principle has set severe limits on the amount of tolerable decoherence for feasible QC operations. More than $10^{4}$ reliable quantum gate operations need to be performed during the coherence time, while the fidelity of gate operation could be lost for too short a gate duration. The spin decoherence in Si:P system has been intensively studied both experimentally and theoretically in recent years[10,11,12,13,14]. Due to the weakness of electron-phonon and spin-orbital interaction, the longitudinal spin relaxation time $T_{1}$ at low temperature can be as long as $10^{3}$ seconds[15], while the transverse spin dephasing time $T_{2}$ is of the order of $60 \mathrm{~ms}$ at $7 \mathrm{~K}$ for isotopically purified ${ }^{28} \mathrm{Si}: \mathrm{P}$, as detected by spin echo technique[11]. Theoretically it is expected that $T_{2}$ can be as long as $2 T_{1}$ in isotopically purified ${ }^{28} \mathrm{Si}$. This extraordinarily long decoherence time is another attractive aspect of spin-based Si:P QC architectures.

Due to the attractive features of the Si:P QC, recently there have been much increased research activities on Si:P donors, such as the re-estimate of spin decoherence time[10-14], the stark effect and gate-induced ionization of single donor[16-18], and most importantly, the exchange coupling for a donor pair[19-24].

The present paper is organized as follows. In the next section we review the shallow donor problem in $\mathrm{Si}$, incorporating the details of the $\mathrm{Si}$ band structure. We present the necessary formalism for the multi-valley effective mass equation for single donor and the unrestricted Hatree-Fock approach with generalized valence bond(GVB) wave functions for coupled donor pair. We study in Section III the energy level splitting and charge distribution for a quantum-well-confined single donor. Section IV presents the most important results of our work. The exchange coupling for a donor pair is extensively studied, including the effect of quantum well size, the oscillatory behavior v. s. donor position shift, and gate voltage dependence. The calculation of the realistic gate potential is presented in the appendix by solving the Poisson equation with appropriate boundary conditions. In the last section we summarize our work and present relevant remarks on the Si:P based QC architecture. 


\section{CHARACTERIZATION OF PHOSPHOROUS DONORS IN SILICON}

The problem of impurities in semiconductors was extensively studied in the sixties and seventies of last century, along with the growth of the semiconductor industry [25]. The most common and successful approach to solve the single impurity problem is to utilize the effective mass approximation (EMA) [26]. For semiconductors like silicon, there are several equivalent valleys in the conduction band due to crystal symmetry, and the original hydrogenic EMA needs to be modified to incorporate the interactions between degenerate valleys known as "valley-orbit coupling". The main contribution to the valley-orbit coupling arises from the short-range core part of the impurity potential. The impurity potential is usually not Coulombic near the core, and it is difficult to calculate it from first principles due to the complication of exchange and correlation effects between the donor electron and core electrons. Therefore, in the early applications of EMA, the agreement between theories and experiments is good for excited states but poor for ground states which have a high probability inside the core. Later on many theoretical attempts [27] were made to account for the discrepancies between the hydrogenic binding energies $E_{H}$ and the observed donor binding energy, $E_{0}$. This difference, mainly due to the greatly reduced of screening of Coulomb potential inside the so-called "central-cell" region, is commonly referred to as the "chemical shift" or "central-cell correction".

Experimentally [28], for phosphorous donors in bulk silicon, the zero-field binding energies are $E_{b}+\Delta_{0}=45.59 \mathrm{meV}$ (the nondegenerate ground state with A symmetry), $E_{b}+\Delta_{0}-\Delta_{E}=$ $33.89 \mathrm{meV}$ (two-fold degenerate state with E symmetry), and $E_{b}+\Delta_{0}-\Delta_{T}=32.58 \mathrm{meV}$ (three-fold degenerate state with $T_{2}$ symmetry)V. Here $E_{b}$ is the binding energy of six-fold degenerate ground states obtained by single-valley EMA. Kohn and Luttinger's theory [26] yield $E_{b} \approx 29 \mathrm{meV}$, while the more elaborate work by Faulkner [29] gives $E_{b} \approx 31.27 \mathrm{meV}$. $\Delta_{0}, \Delta_{E}, \Delta_{T}$, known as central-cell corrections, are positive, and in contrast to $E_{b}$, depend on the particular impurity species. For Si:P donors, $\Delta_{0} \approx 16.6 \mathrm{meV}$ according to Kohn and Luttinger (or $14.3 \mathrm{meV}$ according to Faulkner), $\Delta_{E} \approx 13.0 \mathrm{meV}$ and $\Delta_{T}=11.7 \mathrm{meV}$.

It is pointed out by Pantelides and Sah [30] that the concept of chemical shifts or centralcell corrections are ill-defined in the one-valley EMA framework, except for isocoric impurities (where impurity and host have the same number of core electrons, e.g., Si:P donor), and the "chemical shift" must arise almost entirely from intervalley mixing, which causes 
the splitting of the six-fold degenerate ground state level of donors embedded in silicon as observed experimentally. Therefore, a multi-valley effective mass equation (MVEME) is needed to correctly solve the impurity problem in a host with multi-valley conduction band.

The first effective-mass equation for a multi-valley band was obtained by Twose as reported by Fritzsche [31]. For a single substitutional donor situated at $\mathbf{R}$ in silicon, whose band structure has six equivalent valleys, the electron wave function in real space can be written as:

$$
\Phi(\mathbf{r}-\mathbf{R})=\sum_{i} F_{i}(\mathbf{r}-\mathbf{R}) e^{\mathbf{k}_{i} \cdot(\mathbf{r}-\mathbf{R})} u_{i}(\mathbf{r})
$$

where $u_{i}(\mathbf{r})$ is the periodic part of the Bloch functions for silicon lattice. $F_{i}$ is the envelope function for the i-th valley, with $i= \pm x, \pm y, \pm z$ corresponding to six valleys centered at the band minima $\pm k_{0} \hat{\mathbf{x}}, \pm k_{0} \hat{\mathbf{y}}$, and $\pm k_{0} \hat{\mathbf{z}}$, respectively. $k_{0}=0.86 \times 2 \pi / a_{0}$, with $a_{0}=5.43 \AA$ the lattice constant for silicon crystal. The Twose's multi-valley effective mass equation(MVEME) reads:

$$
\sum_{i} \alpha_{i} e^{i\left(\mathbf{k}_{\mathbf{i}}-\mathbf{k}_{\mathbf{j}}\right) \cdot \mathbf{r}}\left[T_{i}(-i \nabla)+U(\mathbf{r})-E\right] F_{i}(\mathbf{r})=0
$$

where the set of coefficients $\alpha_{i}$ is determined by crystal symmetry group. $U(\mathbf{r})$ is the impurity potential and $T_{i}(\mathbf{k})$ is the expansion of the silicon band structure $E^{0}(\mathbf{k})$ at the i-th minimum. For example, around the minimum along $+x$ direction, located at $+k_{0} \hat{x}$, the energy may be expanded as

$$
E^{0}(\mathbf{k}) \simeq \frac{\hbar^{2}}{2 m_{l}}\left(k_{x}-k_{0}\right)^{2}+\frac{\hbar^{2}}{2 m_{t}}\left(k_{y}{ }^{2}+k_{z}{ }^{2}\right) .
$$

where $m_{l}$ and $m_{t}$ are the longitudinal and transverse effective masses, respectively.

The MVEME [Eq. (2)] is essentially a set of coupled equations for the envelope functions associated with different valleys. If the inter-valley mixing, also called valley-orbital interaction, is negligibly small, the envelope functions for different valley nearly coincide and the set of MVEME equations reduce to one single-valley EME. That's the origin of the six-fold degenerate ground state for donors in silicon obtained by Kohn and Luttinger [26]. Only by taking into account the coupling between different valleys we can obtain correct splitting of the ground state. For a substitutional donor in silicon, the impurity potential has tetrahedral symmetry. Valley-orbit coupling mixes the six Bloch functions belonging to different valleys with three kinds of symmetries according to the irreducible representations of the $T_{d}$ group. The linear combinations of them form a singlet with $A_{1}$ symmetry, a doublet with 
$E$ symmetry, and a triplet with $T_{2}$ symmetry. The appropriate linear combinations are

$$
\begin{gathered}
A_{1}:(X+\bar{X}+Y+\bar{Y}+Z+\bar{Z}) / \sqrt{6} ; \\
E:(X+\bar{X}-Y-\bar{Y}) / 2,(2 Z+2 \bar{Z}-X-\bar{X}-Y-\bar{Y}) / \sqrt{12} ; \\
T_{2}:(X-\bar{X}) / \sqrt{2},(Y-\bar{Y}) / \sqrt{2},(Z-\bar{Z}) / \sqrt{2} .
\end{gathered}
$$

Here $X, \bar{X}, Y, \bar{Y}, Z$ and $\bar{Z}$ are the labels for the six Bloch functions, according to the directions of conduction band minima.

Eq. (2) can be solved once we know the form of the impurity potential $U(\mathbf{r})$. For substitutional donors, such as phosphorous donors in silicon, the impurity potential, $U(\mathbf{r})$, consists of two parts: $U_{b}(\mathbf{r})$ and $U_{s}(\mathbf{r})$, where $U_{b}(\mathbf{r})$ is essentially the difference between the potential of an impurity ion and a host ion, and $U_{s}(\mathbf{r})$ arises from the redistribution of valence electrons in the crystal, caused by the presence of $U_{b}(\mathbf{r})$. For phosphorous donors in silicon, the point-charge model is reliable and linear response theory can be validly applied to solve the impurity potential[27,30]. The resulting impurity potential in $\mathrm{k}$ space can be written as

$$
U(\mathbf{q})=U_{b}(\mathbf{q}) / \epsilon(\mathbf{q})
$$

with $U_{b}(\mathbf{q})$ given by the Fourier transform of

$$
U_{b}(\mathbf{r})=-n e^{2} / r+W_{b}(\mathbf{r})
$$

where the first term is the Coulomb potential of one point charge and $W_{b}(\mathbf{r})$ denotes the short-range contribution. The q-dependent dielectric screening function, instead of simply the constant $\epsilon_{0}$ in the hydrogenic impurity potential, is used to take care of the correct screening effect both near and far away from the core. A set of exponential functions may be used to simulate the dielectric screening function[30].

For Si:P donors, $W_{b}$ is very small and localized within about one lattice constant. Similar to the short-range behavior of the dielectric screening function, it's difficult to calculate. Therefore we include all of the short range effects in the modelled dielectric screening function. For convenience we will approximate the dielectric screening effect by a set of isotropic Gaussian functions. It is found that, a single-parameter Gaussian function is adequate for 
simulating the (inverse of) dielectric screening function calculated in Ref. [32] within several lattice constants $\left(a_{0}=5.43 \AA\right.$ ) ) and yield the correct long-range limit. Explicitly, the dielectric function used in this paper has the following form:

$$
\frac{1}{\epsilon(r)}=\frac{1}{\epsilon_{0}}\left[1+\left(\epsilon_{0}-1\right) \exp \left(-\alpha_{c} r^{2}\right)\right]
$$

where $\alpha_{c}$ is treated as an empirical parameter. We find that $\alpha_{c}=1.13 a$.u. gives reasonable agreement with the dielectric function in the range of more than two lattice constants and it yields the correct intervalley splitting of the Si:P ground state.

Eqs. (1), (2), (3) and (7) constitute all we need to adequately describe the electron wave function and energy levels for an isolated Si:P donor. For our specific QC architecture, phosphorous donors are confined in a $\mathrm{Si} / \mathrm{SiGe}$ quantum well. The large in-plane strain presented in the quantum well further reduces the crystal symmetry. As discussed in Refs. [21] and [33] within perturbation theory, the interplay of strain and valley-orbit effect makes only two valleys relevant for the low-lying states. For the quantum well shown in Fig. 1, the minimums of two lowest energy valleys are at $k_{x}=k_{y}=0$ and $k_{z}= \pm k_{0}$. Their energies are well separated from those at the minimums of the other four higher energy valleys by more than $100 \mathrm{meV}$. Thus, only the $+z$ and $-z$ valleys contribute to the ground state. Explicitly, we are left with two kinds of linear combinations of Bloch wave functions,

$$
\begin{gathered}
T_{z}^{+}: \text {symmetric }:(Z+\bar{Z}) / \sqrt{2} ; \\
T_{z}^{-}: \text {antisymmetric }:(Z-\bar{Z}) / \sqrt{2} .
\end{gathered}
$$

The symmetric $T_{z}^{+}$state is the ground state for a single donor at the center of a strained quantum well.

Analogous to Eq. (1), for a single donor located at $\mathbf{R}=0$, the two-valley wave function can be written as

$$
\Phi(\mathbf{r})=F_{+z}(\mathbf{r}) e^{+k_{0} \cdot z} u_{+z}(\mathbf{r})+F_{-z}(\mathbf{r}) e^{-k_{0} \cdot z} u_{-z}(\mathbf{r})
$$

The corresponding two-valley effective mass equation with external field is given by

$$
\begin{gathered}
{\left[T_{+z}(-i \nabla)+U(\mathbf{r})+V_{e x t}-E\right] F_{+z}(\mathbf{r})+} \\
e^{+i 2 k_{0} z}\left[T_{-z}(-i \nabla)+U(\mathbf{r})+V_{e x t}-E\right] F_{-z}(\mathbf{r})=0
\end{gathered}
$$




$$
\begin{aligned}
& e^{-2 i k_{0} z}\left[T_{+z}(-i \nabla)+U(\mathbf{r})+V_{e x t}-E\right] F_{+z}(\mathbf{r}) \\
& +\left[T_{-z}(-i \nabla)+U(\mathbf{r})+V_{e x t}-E\right] F_{-z}(\mathbf{r})=0
\end{aligned}
$$

where $T_{+z}=T_{-z} \equiv T_{z}$ has an operator form as follows:

$$
T_{z}(-i \nabla)=-\frac{\hbar^{2}}{2 m_{l}} \frac{\partial^{2}}{\partial z^{2}}-\frac{\hbar^{2}}{2 m_{t}}\left(\frac{\partial^{2}}{\partial x^{2}}+\frac{\partial^{2}}{\partial y^{2}}\right) .
$$

The envelope functions for the two valleys, $F_{+z}(\mathbf{r})$ and $F_{-z}(\mathbf{r})$, are coupled together, as shown in Eq. (10). We do not assume the equivalence of $F_{+z}(\mathbf{r})$ and $F_{-z}(\mathbf{r})$ as, e.g., in Refs. [4] and [21], since we shall consider the situation where the system lacks reflection symmetry about $z$.

To solve the electron wave functions and energies, we need to choose some well-behaved basis functions to expand the two envelope functions. We modify the set of bases used in our previous paper[20] (in which we study Si:P donors within spherically averaged EMA) to take into account the anisotropy of the silicon band structure and the broken symmetry brought by the external field.

We use a set of two-dimensional(2D) anisotropic Gaussian functions $\left\{\beta_{j}(y, z)\right\}$ to describe the freedoms in $\mathrm{y}$ and $\mathrm{z}$ directions. Two different kinds of Gaussian functions, $\left\{\exp \left[-\alpha_{i}\left(y^{2}+\right.\right.\right.$ $\left.\left.\left.z^{2} / \xi^{2}\right)\right] ; i=1, \ldots, n_{e}\right\}$ and $\left\{z \exp \left[-\alpha_{i}\left(y^{2}+z^{2} / \xi^{2}\right)\right] ; i=n_{e}+1, \ldots, n_{r}=n_{e}+n_{o}\right\}$, are used to simulate the envelope functions with even and odd symmetry (with respect to $z$ ). The optimum value for the anisotropy factor $\xi=0.57$ is obtained by minimizing the ground state energy. We note that both even and odd symmetric bases are necessary when the external potential lacks reflection symmetry with respect to the doping plane $(\mathrm{z}=0)$. The set of Gaussian parameters $\left\{\alpha_{i}\right\}$ are optimized such that a linear combination of the 3D Gaussian functions $\left\{\exp \left(-\alpha_{i} r^{2}\right)\right\}$ best resembles the 1s wave function of a hydrogen atom, while a linear combination of $\left\{z \exp \left(-\alpha_{i} r^{2}\right)\right\}$ best resembles the $2 p_{z}$ orbital.

In our multi-qubit $\mathrm{QC}$ architecture, a line of substitutional donors are arranged along the [100] axis, and neighboring qubits can be coupled together mainly by the wave-function overlaps along the $\mathrm{x}$ axis. To facilitate our calculation and to better describe the inter-donor coupling, we place the system in an artificial 1D box with infinite potential barriers. The center of the box is at $x=0$ and the box size $L$ is chosen to be large enough (at least $10 \mathrm{~nm}$ from the donor ion to either side wall) so that it has negligible effect on the donor 
binding energy and charge distribution. With the introduction of the box, we can use a set of orthogonal sine functions, $\left\{\sqrt{2 / L} \sin \left[\frac{m \pi}{L}(x+L / 2)\right], m=1, \ldots, n_{x}\right\}$, to describe the electron freedom along the $\mathrm{x}$ direction. Again, such a set of functions includes both odd (even $\mathrm{m}$ ) and even (odd $\mathrm{m}$ ) functions of $x$. This is needed when the donor ion is shifted from the box center (which is the case when more than one donor are present) and/or a nonsymmetric external potential is introduced. Combining the set of sine functions and $\left\{\beta_{j}(y, z), j=1, \ldots, n_{r}\right\}$, we get a set of bases:

$$
B_{j m}=\sqrt{2 / L} \sin \left[\frac{m \pi}{L}(x+L / 2)\right] \beta_{j}(y, z)
$$

where $j m=(j-1) \times n_{x}+m$ and $N=n_{r} \times n_{x}$ is the total number of our basis functions.

The suitability of this construction of bases was discussed and verified in a previous publication [20] by comparing our numerical results to exact results in various limits.

Now we can expand the two valley-dependent envelope functions as a linear combination of our bases. We write:

$$
\begin{gathered}
F_{+z}=\sum_{j m=1}^{N} c_{j m} B_{j m} \\
F_{-z}=\sum_{j m=1}^{N} c_{j m+N} B_{j m} .
\end{gathered}
$$

Substituting Eqn. (13) to Eqn. (10), we obtain a matrix equation for the $2 \mathrm{~N}$-dimensional vector $\left\{c_{j}, j=1, \ldots, 2 N\right\}$ :

$$
\sum_{n=1}^{2 N} H_{m n} c_{n}=E \sum_{n=1}^{2 N} O_{m n} c_{n}
$$

where $\left\{H_{m n}\right\}$ and $\left\{O_{m n}\right\}$ are $2 N \times 2 N$ Hermitian matrixes, which can be written as $2 \times 2$ block forms in terms of four $N \times N$ matrixes:

$$
H_{2 N \times 2 N}=\left[\begin{array}{ll}
H^{11} & H^{12} \\
H^{21} & H^{22}
\end{array}\right]
$$

where $H^{11}$ and $H^{22}$ are the intra-valley Hamiltonian matrixes for $+\mathrm{z}$ and $-\mathrm{z}$ valley, respectively, while $H^{12}$ and $H^{21}$ describe the inter-valley coupling. Obviously, $H^{21 *}=H^{12} \equiv H^{D V}$, since $H_{2 N \times 2 N}$ is Hermitian. We also have $H^{11}=H^{22} \equiv H^{S V}$ since we have chosen the same set of basis functions to expand the envelope functions for the two valleys. The decomposition of $O_{2 N \times 2 N}$ has similar properties $\left(O^{21 *}=O^{12} \equiv O^{D V}\right.$ and $\left.O^{11}=O^{22} \equiv O^{S V}\right)$. 
Explicitly, the matrix elements of $H^{S V}$ and $H^{D V}$ are written as

$$
\begin{gathered}
H_{i j}^{S V}=\int d \mathbf{r} B_{i}(\mathbf{r})\left[T_{z}(-i \nabla)+U(\mathbf{r})+V_{e x t}\right] B_{j}(\mathbf{r}) ; \\
H_{i j}^{S D}=\int d \mathbf{r} B_{i}(\mathbf{r})\left[T_{z}(-i \nabla)+U(\mathbf{r})+V_{e x t}\right] B_{j}(\mathbf{r}) e^{i 2 k_{0} z} .
\end{gathered}
$$

where it is understood that the operators inside the square brackets only act on the envelope basis functions but not on the valley phase factor. $O^{S V}$ and $O^{D V}$ can be obtained by replacing $[\cdots]$ in Eq. (16) by 1.

Our main equation, Eq. (14), is a generalized eigenvalue equation composed of a $2 N \times 2 N$ hamiltonian matrix $\mathrm{H}$ and a $2 N \times 2 N$ overlap matrix. Since the sine functions in $x$ are already orthogonal, we only need to perform the Gram-Schmidt orthogonalization process on the set of $y, z$ dependent gaussian functions to convert the generalized eigenvalue equation into a standard eigenvalue equation. We have

$$
\tilde{H}^{s} \tilde{C}=E \tilde{C}
$$

where $\tilde{H}^{s}$ is a $2 N \times 2 N$ Hermitian matrix and $\tilde{C}$ denote a 2 N-dimensional eigenvector. The full single donor electron wave function can be constructed from $\tilde{C}$ according to Eqs. (9) and (13), once the Bloch functions $u_{ \pm z}(\mathbf{r})$ are known.

To study a donor pair coupled by mutual Coulomb interaction, we need to build the appropriate form of the two-electron wave function and the effective two-electron Hamiltonian(Born-Oppenheimer approximation is implicitly assumed).

Like the potential term in the one-electron MVEME, the electron-electron(e-e) interaction term should also include contributions from different valleys. However, for well-separated donors, the short-range effect on the mutual Coulomb interaction is negligible. Therefore, the e-e interaction takes a simple form,

$$
U_{e e}\left(\mathbf{r}_{1}, \mathbf{r}_{2}\right)=\frac{1}{\epsilon_{s}\left|\mathbf{r}_{1}-\mathbf{r}_{\mathbf{2}}\right|}
$$

where $\epsilon_{s} \equiv \epsilon(0)=11.4$ is the static dielectric constant.

The two-electron Hamiltonian can thus be written as

$$
H\left(\mathbf{r}_{1}, \mathbf{r}_{2}\right)=\tilde{H}^{s}\left(\mathbf{r}_{1}\right)+\tilde{H}^{s}\left(\mathbf{r}_{\mathbf{2}}\right)+U_{e e}\left(\mathbf{r}_{1}, \mathbf{r}_{2}\right) .
$$

For a well-separated donor pair, the two-electron spatial wave function with the lowest energies can be constructed from the one-electron orbits of both donors, according to the 
Heitler-London approximation. The lowest two-electron state is a singlet in the absence of magnetic field and is a symmetric combination of one-electron wave-function products, while the second lowest two-electron state is a triplet and antisymmetric with respect to the interchange of spatial indexes of the two electrons. Explicitly, we write

$$
\Psi_{ \pm}\left(\mathbf{r}_{1}, \mathbf{r}_{2}\right)=\frac{1}{\sqrt{2\left(1+S^{2}\right)}}\left[\Phi_{L}\left(\mathbf{r}_{1}\right) \Phi_{R}\left(\mathbf{r}_{2}\right) \pm \Phi_{R}\left(\mathbf{r}_{1}\right) \Phi_{L}\left(\mathbf{r}_{\mathbf{2}}\right)\right]
$$

where the subscript $+(-)$ denotes the singlet (triplet) state, $\Phi_{L}\left(\Phi_{R}\right)$ denotes a one-particle wave function localized at the left (right) donor site, and $S \equiv\left|\left\langle\Phi_{L} \mid \Phi_{R}\right\rangle\right|$ the wave function overlap.

Our goal is to solve the Schrödinger equation

$$
H\left(\mathbf{r}_{1}, \mathbf{r}_{2}\right) \Psi_{ \pm}\left(\mathbf{r}_{1}, \mathbf{r}_{2}\right)=E_{ \pm} \Psi_{ \pm}\left(\mathbf{r}_{1}, \mathbf{r}_{2}\right)
$$

The Heisenberg exchange coupling strength, J, which plays an essential role for a lot of solid-state QC proposals, is given by the energy difference of the lowest singlet and triplet states: $J=E_{-}-E_{+}$.

As we did successfully before [20], we use the unrestricted Hartree-Fock method to solve the lowest singlet and triplet states in the form of Eq. (20). Let the expansion coefficients in terms of the orthogonalized bases $\left\{\tilde{B}_{n}(\mathbf{r}) ; n=1, \ldots, 2 N\right\}$ for $\Phi_{R}$ and $\Phi_{L}$ be $R_{n}$ and $L_{n}$, respectively. Assume that in a given iteration, we have already known the expansion coefficients $R_{n}$ for $\Phi_{R}(\mathbf{r})$. The above two-particle eigen-value equation can be reduced to a single-particle eigen-value problem by projecting it into the state $\Phi_{R}$. The projected eigen-value equation within the basis now reads

$$
\begin{gathered}
\sum_{n}\left[\left\langle n^{\prime}\left|H^{s}\right| n\right\rangle+\left\langle\Phi_{R}\left|H^{s}\right| \phi_{R}\right\rangle \delta_{n^{\prime}, n} \pm\left\langle n^{\prime}\left|H^{s}\right| \Phi_{R}\right\rangle R_{n}\right. \\
\left. \pm R_{n^{\prime}}\left\langle\Phi_{R}\left|H^{s}\right| n\right\rangle+\left\langle n^{\prime}, \Phi_{R}\left|U_{e e}\right| n, \Phi_{R}\right\rangle \pm\left\langle n^{\prime}, \Phi_{R}\left|U_{e e}\right| \Phi_{R}, n\right\rangle\right] L_{n} \\
=E \sum_{n}\left(\delta_{n^{\prime}, n} \pm R_{n^{\prime}} R_{n}\right) L_{n} .
\end{gathered}
$$

Thus, $\Phi_{L}(\mathbf{r})$ can be solved via the standard diagonalization procedure within the one-particle basis. The newly obtained $\Phi_{L}(\mathbf{r})$ is then used to solve the new $\Phi_{R}(\mathbf{r})$, and we do it iteratively until the singlet and triplet ground state energies and wave functions converge. 
As we discussed and examined in our previous paper [20], the above method dealing with a two-particle problem, which we call UHF with generalized valence bond wave functions, does a better job than the usual single-determinant HF method, or the HL approximation. The floating-phase Heitler-London approach, proposed by Koiller et. al. [23], corresponds to a special case of our method where a single parameter describing the relative phase of $\Phi_{L}$ and $\Phi_{R}$ is allowed to vary to improve the naive application of the usual HL approximation. Compared to the configuration interaction(CI) method, which uses a set of two-particle wave function bases(each basis corresponding to a Slater determinant) to do an exact diagonization, our proposed method uses 2 relaxed determinants to expand the wave function. However, we adopt our method instead of the CI method because it can handle the wellseparated two-donor problem adequately and requires much less computational resources, while the large number of bases needed in the CI method makes it currently unpractical.

For convenience we define the effective Bohr radius

$$
a_{B}^{*}=\frac{\epsilon_{s} \hbar^{2}}{m^{*} e^{2}}=0.529 \stackrel{\AA^{\prime}}{m^{*} / m_{0}} \simeq 23.4 \AA
$$

and the effective Rydberg (donor binding energy)

$$
R y^{*}=\frac{e^{4} m^{*}}{2 \hbar^{2} \epsilon_{s}^{2}}=13.59 \mathrm{eV} \frac{m^{*} / m_{0}}{\epsilon_{s}^{2}} \simeq 27 \mathrm{meV} .
$$

where $m_{0}$ is the free electron mass, $m^{*}$ is the spherically averaged effective mass, $m^{*}=$ $\left(\frac{1}{3} m_{l}^{*-1}+\frac{2}{3} m_{t}^{*-1}\right)^{-1}=0.258 m_{0}$ with $m_{l}=0.911 m_{0}$ and $m_{t}=0.190 m_{0}$ for silicon.

Throughout the paper, if not specified, we will use the effective atomic units (a.u.) in which distance is measured in $a_{B}{ }^{*}$ and energy measured in $R y^{*}$.

\section{SINGLE PHOSPHOROUS DONOR IN SILICON QUANTUM WELL}

In this section we will study the single $\mathrm{Si}: \mathrm{P}$ donor confined in a Si/SiGe quantum well with the method we developed in Section II. Although there have been enormous publications in the past on the theory of isolated donors in silicon [27], only in recent years have silicon donors been studied in a general, inhomogeneous environment due to its relevance to gate control of the bounded electrons in quantum computing context. The present authors [20] studied the effects of a uniform electric field and a one-dimensional parabolic external potential on a pair of Si:P donors, within one-valley spherical EMA. Martins et. al. [34] 
addressed the behavior of shallow donors in silicon under a uniform electric field, using a tight-binding model. Smit et. al. analyzed in Ref. [16] the effect of a small nearby gate on a hydrogenlike impurity in a semiconductor up to field ionization and in Ref. [18] the small electric field dependence of the lowest energy states in donors and acceptors, beyond the single-vally EMA, by applying symmetry arguments and perturbation theory. Blom et.al. [33] used a one-valley hydrogenic effective-mass model with central-cell corrections for calculating the properties of shallow donors inside or outside heterostructure quantum wells. Very recently, Friesen [17] developed a multi-valley effective mass theory to study the Stark effect of substitutional donors in silicon, but with valley-orbit coupling treated perturbatively.

For shallow donors in a stressed $\mathrm{Si} / \mathrm{SiGe}$ quantum well, the lattice-constant mismatch lifts the degeneracy of the silicon conduction band and only the two lowest-energy $(+z$ and -z) valleys are populated. The strain-induced splitting causes the same amount of energy shift for the left two valleys, so we assume its effect is completely included in the band offset $U_{s}$.

The external field potential in Eq. (10) is thus composed of two parts: the square QW potential $V_{Q W}$ and the gate potential. Details for calculating the gate potential profile can be found in Appendix.

The quantum well potential has the following form:

$$
V_{Q W}(z)= \begin{cases}U_{s} & \text { if }\left|z-z_{m}\right|>W / 2 \\ 0 & \text { otherwise }\end{cases}
$$

where $W$ is the width of the quantum well and $z_{m}$ the position of the QW central plane.

First we study the case where only $V_{Q W}$ is present (without the gate potential). We solved the two-valley EME for a single donor placed in the middle of the well, with the QW width varied from $4 \mathrm{~nm}$ to $16 \mathrm{~nm}$. The single donor atom is also at the center of the artificial box. To study the lowest energy states, we only need to consider bases with even symmetry with respect to both $\mathrm{x}$ and $\mathrm{z}$. Ten Gaussian functions and 120 plane wave functions are used to construct the singe-particle bases. The single parameter in the dielectric function, $\alpha_{c}$, if taken as 1.13 a.u., yields a intervalley splitting of $3.2 \mathrm{meV}$ between the ground state and the first excited-state, when the QW width is as large as $16 \mathrm{~nm}$. Note that the ground state energy in wide well limit is approximately $-34.21 \mathrm{meV}$, close to the experimental value 
of the $1 \mathrm{~s}$ states with $T_{2}$ symmetry for a phosphorous donor in bulk silicon. The value of intervalley splitting for a single $\mathrm{P}$ donor in a strained Si quantum well, although not available experimentally, was estimated to be $3.3 \mathrm{meV}$ in large strain limit by Koilter et. al. [21] within perturbation theory, while Blom et. al. [33] estimated the central-cell shifts by relating it to the bulk shifts with symmetry consideration. The shift of the lowest two states from the QW one-valley effective-mass value is given by [33]:

$$
\frac{\left|\Psi_{Q W}\left(\mathbf{r}_{0}\right)\right|^{2}}{\left|\Psi_{1 s}\left(\mathbf{r}_{0}\right)\right|^{2}}\left[-\Delta_{0}+\left\{\begin{array}{l}
2 \Delta_{E} / 3 \\
\Delta_{T}
\end{array}\right\}\right] .
$$

where $\left|\Psi_{Q W}\left(\mathbf{r}_{0}\right)\right|^{2}$ and $\left|\Psi_{1 s}\left(\mathbf{r}_{0}\right)\right|^{2}$ are the QW and bulk envelope function amplitudes near the impurity center, respectively. The values of $\Delta_{0}, \Delta_{E}$, and $\Delta_{T}$ are given in Section II, for phosphorous donors in bulk silicon.

In the large QW width limit, the QW-bulk ratio for envelope function amplitudes should be of the order of unity, which gives a valley splitting of about $3 \mathrm{meV}$, consistent with our calculation. Figure 2 shows our calculation of the Si:P ground-state and first-excited-state energies and their splitting as a function of QW width in large scale. We see that both the ground and excited state energies increase as we decrease the QW width, as a consequence of larger kinetic energy for stronger confinement. The intervalley splitting also increases in a similar way. With Eq. (26), Blom et. al. predicted the same behavior for the valley splitting as the QW width is varied. They argued that, the ratio in Eq. (26) is expected to increase as the QW is narrowed, due to additional confinement, therefore, the intervalley splitting should also increase.

This can be verified by inspecting the charge distribution. We plot in Fig 3 (a) and (b) the 1D averaged charge distribution (obtained by integrating out the other two coordinates in the 3D charge density) along z-axis and x-axis, respectively. The inset of Fig. 3(b) shows the $1 \mathrm{D}$ averaged charge distribution along y-axis. First we note that, the curves of $\rho_{x}$ and $\rho_{y}$ are very similar to each other, although $\rho_{x}$ is slightly more spread out, due to different basis functions we use for $x$ and $y$ directions (by symmetry they should coincide with each other). The charge distribution along $\mathrm{z}$ axis oscillates with a period $2 \pi / \Delta k \equiv \pi / k_{0} \approx 3 \AA$, resulting from the full wave function construction in Eq. (9). Due to influence of QW barriers, the charge distribution is more concentrated around the donor nucleus for narrower confinement, as can be seen from the comparison of $\rho_{z}$ for the QW width at $6 \mathrm{~nm}$ and $10 \mathrm{~nm}$. 
To our surprise, the charge distribution in the direction parallel to the QW plane, shown in Fig.3 (b), also shrinks toward the impurity center as the QW is narrowed. This behavior is counterintuitive since we would have expected the shrinking of wave function in one direction would accompany dispersing in other directions. The synchronization of charge redistribution in all the 3 dimensions upon the change of $1 \mathrm{D}$ confinement, is due to the cooperative efforts to maintain the $3 \mathrm{D}$ shape of 1 s orbital so as to lower the ground state energy. We expect that the QW confinement should also have a significant effect on the exchange coupling of two neighboring donor electrons inside the quantum well, as we shall discuss in Sec. IV, since the dispersion of charge distribution would enhance the electron wave function overlap as the QW confinement is reduced.

We plot in Fig. 4(a) the valley splitting $\Delta E_{P}$ of the Si:P ground state as the QW width varies in small scale. The calculated points cover QW width range from about 8nm to 10nm, with a spacing of $a_{0} / 4 \simeq 1.36 \AA$. It clearly shows an oscillatory behavior and the oscillation magnitude decays as the QW width increases, while the oscillation period is approximately one lattice constant $\left(a_{0}\right)$.

For comparison, we also calculate the valley splitting of the lowest QW states, $\Delta E_{W}$. Such a valley splitting has been re-examined recently for $\mathrm{Si} / \mathrm{SiGe}$ heterostructures within a 2-band tight-binding model [35,36]. Here it is done by solving the two-valley EME without the impurity potential. The boundary matching conditions at the QW interfaces are neglected, since the lowest QW states are mostly confined inside the silicon quantum well for a QW width more than 8nm and a barrier height of $300 \mathrm{meV}$. We use the products of 20 (2D)Gaussian functions and 100 sine functions to expand the 3D wave functions of the lowest QW states. Fig 4(b) shows the QW valley splitting for the QW width varing from 50 to 80 , in units of $a_{0} / 4$. First we note that the magnitude of $\Delta E_{W}$ is approximately several tenths of meV for the QW width range shown, consistent with the tight-binding calculation of Ref. [36]. Compared to the valley splitting of impurity states $\Delta E_{P}, \Delta E_{W}$ is about 6 times smaller. This significant difference between the magnitude of $\Delta E_{W}$ and $\Delta E_{P}$ is due to the presence of central-cell correction brought by the impurity potential. Second, similar to $\Delta E_{P}, \Delta E_{W}$ also oscillates and decays as the QW width increases. Comparing the pattern of Fig. 4 (b) with Fig. 4 (a), we see that the oscillation of $\Delta E_{W}$ is much more frequent than $\Delta E_{P}$ on this smallest scale. However, if we view the oscillations at a larger scale (as in Ref. [36]), say, in units of $a_{0} / 2, \Delta E_{W}$ will oscillate less frequent than $\Delta E_{P}$, as shown by 
the dotted lines in Fig. 4 (a) and (b). Note that the oscillation period on the $a_{0} / 2$ scale is about $3.5 a_{0}$, nearly the same as the oscillation shown in Fig. 3 of Ref. [36].

Compared to the sophisticated microscopic tight-binding calculation, we conclude that, our simple calculation, although it has overlooked the delicate boundary matching condition for finite-height barriers, is able to produce similar results. The oscillatory behavior shown in Fig. 4 (a) and (b), is due to the presence of multiple propagating states in the well, which is inherently included in our two-valley effective mass approximation, as shown in the composition of the full wave function in Eq. (9).

We also study the intervalley splitting for the $\mathrm{Si}: \mathrm{P}$ donor states as the donor atom is moved away from the center plane of the quantum well (but still kept in the center of the artificial box). In this case, the reflection symmetry of the QW potential is broken and we need to include both even and odd basis functions with respect to $z$. We use 10 Gaussian functions in the form of $\left\{\exp \left[-\alpha_{i}\left(y^{2}+z^{2} / \xi^{2}\right)\right]\right\}$ (with $\alpha_{i}$ optimized to resemble the 1s orbital of the hydrogen atom [37]) and 6 Gaussian functions in the form of $\left\{z \exp \left[-\beta_{j}\left(y^{2}+z^{2} / \xi^{2}\right)\right]\right\}$ (with $\beta_{j}$ optimized to resemble the $2 p_{z}$ orbital of the hydrogen atom [37]) to take care of the mixed symmetry. Eighty sine functions with even parity is used for the description of freedoms along $\mathrm{x}$ axis.

We plot in the inset of Fig. 4(a) the valley splitting as a function of donor position inside the silicon quantum well with the QW width fixed at $9 \mathrm{~nm}$. The valley splitting also shows an oscillatory behavior as the donor position is shifted. The magnitude of oscillation increases as the donor is moved away from the QW center, while the oscillation shows a double-period structure, with periods $\sim 0.5 a_{0}$ and $\sim 0.75 a_{0}$. The increase of the valley splitting as the donor is moved away from the QW center can be explained with the help of Eq. (26). The QW envelope function amplitude $\left|\Psi_{Q W}\left(\mathbf{r}_{0}\right)\right|^{2}$ is expected to increase as one of the barrier is more easily sensed by the donor bound electron (similar to the case when the quantum well is narrowed).

The effect of gate potential on the single donor will not be presented here, since we focus in this paper on the gate control of exchange coupling for a donor pair, as we shall address in the next section. It is worth mentioning that although the spectrum narrowing is observed (the intervalley splitting decreases as the gate-induced electric field pulls the donor electron away from the central cell), the ground state energy is lowered, in contrast to the result obtained in Ref. [17] for donors in bulk silicon where the Stark effect of phosphorous donor 
in bulk silicon was studied with a perturbative multi-valley effective mass theory.

\section{EXCHANGE COUPLING FOR SI:P DONOR PAIR}

In this section we present systematic studies on a phosphorous donor pair embedded in a strained silicon quantum well. The Heisenberg exchange interaction, defined as the splitting of the lowest singlet and triplet states, plays a central role in most solid state QC architectures. As a result, exchange coupling has been frequently revisited for a pair of localized electrons ever since the first quantum dot QC proposal. Heitler-London approximation is most frequently used, where single-particle orbitals are first variationally determined and two-particle wave function is constructed as in Eq. (20). This kind of approximation, although does fairly well for well separated impurities (or quantum dots), it does not provide enough flexibility to handle the strong-coupling case where electron correlation effect becomes important. As a better alternative, we suggested in Ref. [20] to apply the UHF method with GVB wave function to solve the two-electron system. In this paper we follow this method, combining it with the MVEME, as formulated in Section II.

We consider a pair of coupled donors, labelled by 1 and 2 in Fig. 1. The gate potential to control the exchange coupling is tunable via two gate voltages, $V_{c} \equiv V_{12}$ and $V_{g} \equiv V_{01} \equiv V_{23}$. The gate electrodes right above donor atoms, $V_{1}$ and $V_{2}$ (known as A-gates) are disabled in this paper, since they are not necessary for tuning the exchange coupling for a donor pair (but they will be needed for the cooperation of 3 neighboring donors). The modelling of the electrostatic potential distribution due to applied voltages on the gates is presented in the appendix. The potential distribution in the QW region is give by Eq. (A9). Fig. 5 (a)-(d) shows several gate potential profiles for different values of $V_{c}$ and $V_{g}$. We see that these two gate voltages are indeed capable of tuning the barrier height between two $\mathrm{P}$ donors in the QW.

The width of the artificial $1 \mathrm{D}$ box is set to be the same as the size of the 2-donor unit cell, $L_{x}=4 p$, for the donor array with a spacing of $2 p$. Since the electrons are bound to the donors mainly within $1 a_{B}{ }^{*}$ and dispersed a little in the presence of gate potential, the box boundaries will not affect the charge distribution when $\mathrm{p}$ is larger than $4 a_{B}{ }^{*}$. The box boundaries pass through the centers of gate electrode $V_{01}$ on the left and $V_{23}$ on the right, respectively. $V_{01}$ and $V_{23}$ (cooperating with other gate electrodes) are typically set to 
produce potential barriers high enough to suppress the coupling between the specific donor pair (1 and 2) with neighboring donors (labelled 0 and 3, respectively) during each step of gate operations. In this sense, our $1 \mathrm{D}$ box is no longer artificial. The central J-gate, $V_{12}$, is right above the middle point $(\mathrm{x}=0)$ of the two donors.

First we calculate the exchange coupling when the gate potential is absent $\left(V_{c}=V_{g}=0\right)$. The donor separation, is first set to $R \equiv 2 p=10 a_{B}{ }^{*}$, a generally accepted value for Si:P spin-based QC architecture and within the access of current nano-fabrication technology[38]. We vary the QW width from $4 \mathrm{~nm}$ to $16 \mathrm{~nm}$ and plot the corresponding exchange splitting in Fig. 6. In the wide-well limit, the exchange splitting converges to about $1.4 \times 10^{-6} R y^{*}$, on the same order of magnitude but a little smaller, compared to the exchange coupling for two hydrogenic impurities in bulk silicon estimated via the asymptotic formulae[39]. However, it is about 40 percent larger than the numerically calculated value by single-valley hydrogenic effective mass approximation in Ref. [20]. Our current two-valley calculation is more reliable, since we take into account the effective-mass anisotropy, central-cell correction to the impurity potential, and inter-valley coupling.

We see clearly from Fig. 6 that the exchange splitting J depends strongly on the QW width. It decreases as the quantum well is narrowed. The exchange splitting at $W_{d}=6 \mathrm{~nm}$ is about one order smaller than that at $W_{d}=16 \mathrm{~nm}$. Comparing their single-particle overlaps, $S \equiv\left|\left\langle\Phi_{L} \mid \Phi_{R}\right\rangle\right|$ for singlet states (the overlaps are always zero for triplets according to our construction of two-particle wave functions) with each other, we have $S=5.8 \times 10^{-4}$ for $W_{d}=6 \mathrm{~nm}$ and $S=1.7 \times 10^{-3}$ for $W_{d}=16 \mathrm{~nm}$, respectively. As we narrow the quantum well from $6 \mathrm{~nm}$ to $4 \mathrm{~nm}$ (slightly less than $2 a_{B}{ }^{*}$ ), the exchange splitting is reduced further by one order of magnitude and the overlap $S$ decreases to $2.0 \times 10^{-4}$. This is just what we expected, since from Sec. III (Fig. 3) we know that the charge distribution is shrinking toward the impurity centers in all three dimensions with the increasing of QW confinement. This confinement-induced shrinking effect decreases the overlap between neighboring donor electrons, and therefore, reduces their exchange coupling.

The inset of Fig. 6 illustrates the effect of QW-width variation on small scale on the exchange splitting, where the QW width is varied from 58 to 74 monolayers (approximately from 8 to $10 \mathrm{~nm}$ ) and the spacing of width variation is one monolayer ( 1 monolayer $=a_{0} / 4 \simeq$ $1.36 \AA$ ). Different from the oscillatory behavior of the valley splitting for single donor ground state shown in Fig. 4(a), the exchange splitting varies with the QW width monotonously 
even at the atomic scale.

Now we switch on the gate voltages $V_{g}$ and $V_{c}$. The QW width is fixed at $10 \mathrm{~nm}$, the appropriate value for producing the desired range of the exchange coupling for our $\mathrm{QC}$ architecture. We shall fix the value of $V_{g}$ and vary $V_{c}$ (the central gate voltage), since the potential barrier height between the coupled donors is mainly influenced by voltage difference of the central gate and side gates. Fig. 7(a) shows the influence of the central gate voltage $\left(V_{c}\right)$ on the exchange coupling for donors separated by $10 a_{B}{ }^{*}$, with $V_{g}$ fixed at $-2.0,-1.8$ and $-1.4 \mathrm{~V}$, respectively. We notice that voltage dependence of the exchange splitting can be approximately fitted by an exponential curve (shown as straight line in logarithm-scale plot), similar to our previous simulation where single-valley hydrogenic EMA is employed and the gate potential is modelled by a one-parameter one-dimensional parabolic function. The three lines corresponding to three different values of side gate voltages, are nearly parallel to each other, which means that the logarithmic slope is independent of the gate voltages and it only depends on the intrinsic geometry settings of the QC architecture, such as the donor separation, QW width, and distance of doping plane from the top gates. Therefore, we may define the logarithmic slope as a measure of the gate voltage tunability (with respect to the exchange coupling) as

$$
\eta=\frac{\partial \log J}{\partial V}
$$

where we have omitted the subscript of $V_{c}$ and obviously, $\eta$ has the dimension of $V^{-1}$. Note that in Ref. [40], $\partial J / \partial V$ is similarly defined as the susceptibility of a device to voltage errors. Here, our gate voltage tunability is better defined since it is constant throughout the appropriate working voltage range and it characterizes the intrinsic device properties.

Since $\eta$ is independent of $V_{g}$, we may focus on the case with side gate voltages fixed at $V_{g}=-1.8 \mathrm{~V}$. By tuning the central gate voltage $V_{c}$, we are able to vary the exchange coupling by more than eight orders of magnitude. The full range is not shown in Fig. 7(a), but we can further reduce the exchange splitting to as low as $10^{-11} R y^{*}$ by lowering $V_{c}$ to -3.0 $\mathrm{V}$ for $V_{g}=-1.8 \mathrm{~V}$. The exchange splitting at $V_{g}=-2.1 \mathrm{~V}$ approximately equals the value of $\approx 10^{-6} R y *$ in the absence of gate potential $\left(V_{c}=V_{g}=0\right)$. Reliable quantum computation requires a much smaller J-coupling than the gate-off value for qubit isolation. In Ref. [7], it is argued that the 'off-state' coupling will need to be $J_{\text {off }} \approx 10^{-12} \mathrm{eV}$ or less. We see that the J-coupling at $V_{c}=-3.0 \mathrm{~V}$ can fulfill this requirement.

Furthermore, the large initialization requirement $J_{o n} \approx 200 \mu \mathrm{eV}$ (required to initialize the 
logic 0 state of the 3 -donor qubit)[7] is also within our tunability. At $V_{c}=-0.6(-0.8)$, we can reach a J-coupling of approximately 400(93) $\mu \mathrm{eV}$.

However, as discussed in Ref. [20], we can not arbitrarily enhance the exchange coupling by lowering the potential barrier between donors. To illustrate this, we plot in Fig. 8 the 1D averaged charge distribution along $\mathrm{x}$ axis for the lowest singlet and triplet states at different gate voltages. Each plot shows a two-peak structure with peaks near the donor nuclei. Except for Fig. 8(a) and (d) where the coupling and overlap is weak and the charge distributions for singlet and triplet almost coincide, singlet state has a larger probability than the corresponding triplet state in the central region between donors, due to the Pauli exclusion principle[20]. In Fig. 9(a), although the gate-off exchange coupling is nearly equal to that at $V_{c}=-2.1 \mathrm{~V}$ and $V_{g}=-1.8 \mathrm{~V}$, their charge distributions are different. For the gate-off case, the two charge distribution peaks are symmetrically centered at the donor sites, while for the case of $V_{c}=-2.1 \mathrm{~V}$ and $V_{g}=-1.8 \mathrm{~V}$, they are more spread out and become nonsymmetric with respect to the donor locations. However, the averaged donor electron distances for these two cases, are nearly the same, which is the reason that they have close magnitudes in exchange coupling. Fig. 8(d) shows the charge distribution for the 'offstate' configuration. The peak centers are shifted significantly away from each other with a negligible overlap, and are more dispersed than those in Fig. 8(a). For the case of $V_{c}=-0.8$ shown in Fig. 8(b), the singlet is only slightly different from the triplet, with slightly larger (smaller) weight in the central (donor site) region, and the peak centers remain close to the donor locations as in the case of configurations of (a), although they are pulled toward each other to give an enhanced overlap. In Fig. 8(c), when the (attractive) gate potential becomes even stronger, the difference between singlet and triplet charge distributions becomes more significant, and the weights in the central region are comparable to those in the donor regions. For this case, the gate potential is strong compared to the impurity potential, and electrons have a large probability to be trapped in the central region. This situation is undesirable for QC implementation, since it may complicate the spin dynamics for gate operations, and more importantly, produce extra decoherence channels and reduce gate fidelity. The $V_{c}=-0.8$ configuration is relatively much safer and can nearly produce the desired exchange coupling in the large limit.

In Fig. 7(b), we compare the gate voltage dependence of J-coupling for a pair of donors separated by $\mathrm{R}=8,10$, and $12 a_{B}{ }^{*}$, respectively. First, we notice that for $\mathrm{R}=8$ and $12 a_{B}{ }^{*}$, 
both the corresponding curves deviate (but not significantly) from straight lines on logarithmic scale. The three lines are crossed with each other near $V_{c}=-0.7 \mathrm{~V}$. The logarithmic slopes, indicting the gate voltage tunability on the exchange coupling, are remarkably different. As the central gate voltage is tuned from -2.2 to $-0.6 \mathrm{~V}$, the variation range of exchange coupling is 2, 5, and 9 orders, for $\mathrm{R}=8,10$ and $12 a_{B}{ }^{*}$, correspondingly. Obviously, larger donor separation corresponds to large gate tunability on exchange coupling. However, as we will see below, larger gate tunability also leads to more stringent requirement for voltage control accuracy.

With a time-dependent voltage pulse, the exchange coupling which controls the spin dynamics varies accordingly as the time progresses, and spin states can be manipulated in a controllable way. As a prototype for gate operation, we consider the adiabatic $\sqrt{S W A P}$ operation for two neighboring donor electrons, implemented with a voltage pulse $V_{s}(t)$. In principle, the pulse shape of $V_{s}(t)$ can be arbitrary, although the accumulated phase by the exchange coupling must satisfy the following relation:

$$
\int_{\tau_{s}} d t J(V(t)) / \hbar=\pi / 2 .
$$

Here $\tau_{s}$ is the gate operation time and the exchange coupling is dependent analytically on the central gate voltage via

$$
J(t) \simeq \alpha \times \exp (\eta V(t))
$$

where $\alpha=0.224 \mathrm{eV}$ and $\eta=6.9 \mathrm{~V}^{-1}$ for $R=10 a_{B}{ }^{*}$ and $V_{g} \equiv-1.8 \mathrm{~V}$.

To get a realistic estimate of the gate operation time, we assume a symmetric linear (central gate) voltage pulse, defined by

$$
V(t)= \begin{cases}V_{0}+2 \Delta V\left(t / \tau_{s}+0.5\right) & \text { if }-\tau_{s} / 2 \leq t \leq 0 \\ V_{0}-2 \Delta V\left(t / \tau_{s}-0.5\right) & \text { if } 0 \leq t \leq \tau_{s} / 2\end{cases}
$$

where the voltages during the whole gate operation span a range of $\left[V_{0}, V_{0}+\Delta V\right]$. We have already shown that $[-3.0,-0.8]$ is an appropriate voltage range for $R=10 a_{B}{ }^{*}$, which can give a sufficiently small 'off-state' exchange coupling and large 'on-state' exchange coupling without disturbing the two-electron charge distribution significantly. The maximum exchange coupling is achieved right at the middle of gate operation, corresponding to $V_{0}+\Delta V$. 
Plugging Eqs. (29) and (30) into Eq. (28) with $V_{0}=-3.0 \mathrm{~V}$ and $\Delta V=2.2 \mathrm{~V}$, the gate operation time is found to be $t_{s} \simeq 0.2 \mathrm{~ns}$. Compared to the experimental transverse decoherence time of $T_{2} \approx 60 \mathrm{~ms}[11]$, the proposed pulse gives a relative error of $\sim 10^{-7}$, well satisfying the fault tolerant computation requirement of a relative error less than $10^{-4}$. On the other hand, the gate operation time is restricted from below by the adiabatic gating requirement[41],

$$
\tau_{s}>\tau_{\min }:=\max \left\{\hbar / \delta \epsilon_{1}, \hbar / \delta \epsilon_{2}\right\}
$$

where $\delta \epsilon_{1}$ and $\delta \epsilon_{2}$ are the single-particle lowest level spacing and two-particle lowest singlet level spacing, respectively. $\delta \epsilon_{2}$ is roughly determined by the difference of the on-site and inter-site Coulomb interaction. As discussed in Ref.[41], for ultra small quantum dots, $\delta \epsilon_{1}$ is usually much larger than $\delta \epsilon_{2}$. However, this is not the case for multi-valleyed semiconductors such as Si. The presence of gate potential can cause single-particle spectrum narrowing as discussed in the last section. $\delta \epsilon_{1}$, given by the intervalley splitting of the single donor ground state, is approximately $3.0 \mathrm{meV}$ when gate potential is absent, while with gate potentials at $V_{c}=-0.8 \mathrm{~V}$ and $V_{g}=-1.8 \mathrm{~V}$ we have $\delta \epsilon_{1} \sim 0.43 \mathrm{meV}$. In comparison, $\delta \epsilon_{2}$ is $\sim 0.78 \mathrm{meV}$ at the same voltages. We find that when $V_{g}$ is fixed at $-1.8 \mathrm{~V}$ and $V_{g}$ varies in the voltage range [-3.0,-0.8], $V_{c}=-0.8 \mathrm{~V}$ corresponds to the smallest value for both $\delta \epsilon_{1}$ and $\delta \epsilon_{2}$. Therefore, according to Eq. (31), we get an estimate of the gate operation time, $\tau_{s}>1.5 p s$ on the adiabatic lower restriction. This requirement is also well satisfied by our proposed linear voltage pulse with a gate operation time of $0.2 \mathrm{~ns}$.

This gate-induced narrowing of single-particle level spacing requires an operating temperature to be about six times lower than the gate-off estimate $(\sim 30 K)$, to avoid unwanted thermal transition. To improve the operating temperature, we may increase the contribution of the other four valleys by reducing the Ge composition in the SiGe barrier (i.e. reducing the in-plane strain), decrease the quantum well width, or re-design the gate potential profile. On the other hand, the increase of single-particle level spacing or valley splitting, requiring the single-particle wave functions to be more localized around the impurity centers, would unavoidably reduce the available maximum exchange coupling between neighboring donor electrons(if the donor separation keeps unchanged). Thus the temperature requirement further complicates the issue for optimal design of the QC architecture. However, this problem may be circumvented by the recently proposed multilevel encoding scheme, where a logic qubit is encoded by a subspace of multiple physical levels. The full quantum-computational 
fidelity is maintained in the presence of mixing and decoherence within logical subspaces. For details of multilevel encoding, see Ref. [42].

The gate voltages that control exchange couplings between neighboring donor electrons cannot be produced at arbitrary accuracy. This results in deviation from the desired pulse shape and exchange coupling, causing gate errors. The instantaneous relative error rate can be quantified by $\delta J(t) / J(t)$ and the accumulated error rate $1-F=\int_{\tau_{s}} \delta J(t) d t /(\pi \hbar / 2)$ indicates the loss of gate operation fidelity.

Due to the exponential dependence of exchange coupling on the gate voltages, the instant error rate can be simply related to the gate tunability and voltage fluctuation by

$$
\delta J(t) / J(t)=\eta \delta V(t) .
$$

With this relationship we can obtain a lower bound of the gate fidelity:

$$
F_{-}=1-\eta \delta V_{0},
$$

where $\delta V_{0}$ is the maximum magnitude of voltage uncertainty or fluctuation during the switching time, limited from below by the available control electronics. The other parameter affecting the gate fidelity, is the gate tunability $\eta$ intrinsic to the $\mathrm{QC}$ architecture. We note that, to reduce the loss of fidelity, $\eta$ should not be too large. This restriction, combining with the requirement of enough tunability so as to achieve the desired 'initialization' and 'off-state' exchange coupling, calls for an appropriate parameter zone (such as the donor spacing, the distance of doping plane to the surface gates, and the width of the silicon quantum well) for the realistic quantum computer device design.

For the typical $\eta \sim 7$ for two donors separated by $10 a_{B}{ }^{*}$, a voltage control accuracy of at least $1.4 \times 10^{-5} \mathrm{~V}$ is required to meet the error threshold ( 1 accumulated error in $10^{4}$ operations) of fault tolerant computation. This voltage accuracy is about the same order as in Ref. [4], where the authors estimated it with a flattop exchange coupling pulse for the SiGe quantum dot QC architecture.

Koilter et. al. [19] analyzed the effect of donor position shift on exchange splitting for a well-separated donor pair in bulk silicon, with the Kohn-Luttinger envelope wave function and within Heiter-London approximation. They pointed out the importance of precise donor positioning in $\mathrm{QC}$ implementation, due to the exchange oscillation caused by inter-valley interference inherent in silicon band structure. Later on, a few authors confirmed this kind 
of exchange oscillation under the perturbations due to applied strain [21] or gate fields [22], still within standard HL framework. Recently, a reconfirmation has been done with a floating-phase Heitler-London approach [23]. A similar approach was used for studying $\mathrm{Si} / \mathrm{SiGe}$ quantum dots in Ref. [43]. In that paper, the authors demonstrated that, with strong confinement potential and large in-plane strain, there is no atomic-level oscillations in the single-electron wave function, and consequently no exchange oscillation, in contrast to donors in bulk silicon.

The phosphorous donors in our QC architecture are embedded in a strained Si quantum well with no additional lateral confinement, thus lying between the two extreme cases: donor electron in bulk silicon and electron confined in gated Si/SiGe quantum dots. Furthermore, as we pointed out in Sec II, our approach to the two-localized-electron problem is more advanced than the Heitler-London approximation or its modified version. Now we examine whether the donor-position dependent exchange oscillation exists with and without the presence of gate potential (and with four valleys suppressed by the in-plane strain).

First we study the case where two donors are shifted along $\mathrm{z}$ axis from the QW central plane by the same amount with their separation remaining unchanged. The calculating points are spaced by $a_{0} / 4$, the smallest atomic spacing along $\mathrm{z}$ axis. It is worth mentioning that, in Ref. [19], donor positions are shifted in units of $10 \%$ of the nearest-neighbor distance in silicon lattice, which is nonphysical for substitutional phosphorous donors.

In Fig. 9(a), we plot the exchange coupling versus shift of donor position (up to $3 a_{0}$ ) along the [001] direction. The system is symmetric with respect to the QW central plane in the absence of gate potential, and both donors remain on the [100] axis with their separation fixed at $10 a_{B}{ }^{*}$. We see from Fig. 9(a) that, the exchange coupling does oscillate as both donors are shifted along $\mathrm{z}$ axis, contrary to the monotonous behavior shown in the inset of Fig. 6, where the QW width is varied on atomic scale. The oscillation magnitude is largest as the donors are shifted from the QW center by a single unit $\left(a_{0} / 4\right)$ and it decays as donors are moved away from the center plane. The upper-bound of the $\Delta J / J$ for the smallest displacement, is about $5 \sim 6 \%$. As can be seen from the trend of Fig. 9(a), the exchange coupling tends to increase as both donors are shifted away. The inset shows the corresponding singlet overlaps, similar to the pattern of exchange coupling.

We plot in Fig. 9(b) the exchange coupling as a function of donor position shift when a gate potential is switched on. We find that, the exchange oscillation still exists, but the 
over-all exponentially growing behavior is preserved.

Now we study the exchange coupling as both donors are shifted along their coupling direction, i. e., [100] axis. The smallest possible donor position shift (and donor separation change) is $a_{0} / 2$, to keep both donors in the QW central plane. To better describe the delicate change of the wave function overlap upon position shift along $x$ axis, we use 280 since function(for x-direction freedom and 3 Gaussian functions (for $y$ and $z$ ) to construct the single-particle basis. The parameters in the 3 Gaussians, are adjusted to take care of the central cell correction so that correct valley splitting is reproduced for the single donor ground state. The $1 \mathrm{D}$ box width is set to be $25 a_{B}{ }^{*}(\sim 60 \mathrm{~nm})$, to further reduce possible artificial effects of box boundaries. We plot in Fig. 10 the exchange splitting for donor separation varied from 70 to $90 a_{0} / 2$ (or 190 to $244 \AA$ ). The figure is plotted in logarithmic scale, as the exchange splitting has an exponential dependence on donor separation. The dotted line is an exponential fitting curve. We find that the fitting works fairly well. Compared to the exchange oscillation in Fig. 9(a), the exchange splitting is almost monotonically increasing as the donor separation is reduced. Only for separation larger than $85 a_{0} / 2$, exchange splitting is no longer monotonic and residual oscillation is present. This behavior can be qualitatively understood in the following way. The valley-interference induced exchange oscillation is analogous to noise, which is prominent when the exchange coupling (analogous to signal) is weak. The exponential dependence of exchange splitting on donor separation, tends to suppress the oscillation for small enough separation (strong enough exchange coupling). If we take the exponential fitting curve as a reference, the deviation oscillates strongly from both sides of the reference curve at large separation and decays as separation is decreased.

Based on our calculation, it is worth to make a few remarks on the importance of precise donor positioning to fault tolerant quantum computation. First, the deviation of donors from any host atom site is highly undesirable, since the electronic properties of an interstitial donor is remarkably different from a substitutional donor. Second, a recent report shows that the STM-based lithographic technology can position single P atoms into Si with $\sim 1 \mathrm{~nm}$ accuracy[44]. If unfortunately, a donor atom is shifted away from the desired site, it will result in large computation error (due to the deviation of exchange coupling from desired value) and qubit measurement error (due to the qubit leakage to undesired subspace). However, this should be able to be detected in the calibration process described in Ref. [7], and we can either adjust the gate voltage to reduce error or reject the erroneous qubit. 


\section{SUMMARY AND CONCLUDING REMARKS}

In this paper we have presented a systematic study on coupled phosphorous donors in a strained silicon quantum well, relevant for silicon-based quantum computer design. Following the multi-valley effective mass approach, we have developed a two-valley equation for the single donor. By turning the valley-coupled Schödinger equation into a single matrix eigenvalue problem, we are able to solve the donor electron ground state energies and wave functions. The central cell correction is consistently incorporated by our model potential, yielding the correct valley splitting.

We have studied the effects of quantum-well confinement and donor position shift on the valley splitting and charge distribution for the single donor ground state. We find that, as the quantum well is narrowed, the electron wave function shrinks and larger valley splitting is obtained. For a QW at fixed width, the valley splitting also increases as the donor approaches the edge of the QW. We have examined the effects of QW width and donor position variations at the atomic scale. The oscillation of valley splitting for donor electron ground state is observed for both cases. The valley splitting of the lowest QW states is also calculated as a function of QW width and oscillation behavior is confirmed. We have analyzed these results and compared them to relevant papers by other authors.

The unrestricted Hartree-Fock method with GVB wave functions is formulated and applied to a pair of coupled donors, without losing the delicate nature of single-particle wave functions. The advantages of this method over other approaches are discussed. Accurate wave functions and energies of the lowest singlet and triplet states can be obtained. The Heisenberg exchange coupling is calculated at different QW widths and we find that a wider quantum well gives rise to larger exchange splitting. The QW width of $10 \mathrm{~nm}$ is appropriate for our proposed QC design.

We have calculated the gate potential profile numerically by solving Poisson equation with appropriate boundary conditions. The effect of gate voltages on exchange coupling between neighboring donors is analyzed, for several donor separations. We find the appropriate separation and voltage zones suitable for qubit initialization and gate operation. A gate tunability is defined to characterize the efficiency of gates to alter the exchange coupling. With a linear time-dependent voltage pulse shape, we have evaluated the appropriate gate

operation time of $0.2 \mathrm{~ns}$ for a $\sqrt{S W A P}$ operation, considering both the requirements of fault 
tolerant quantum computation and adiabatic operation. An analytic formulae for the lower bound of the gate operation fidelity is obtained, by relating it to the gate tunability and voltage control accuracy. For a typical gate tunability, and to realize fault tolerant quantum computation, a voltage control accuracy of $1.4 \times 10^{-5} \mathrm{~V}$ is necessary.

The valley degeneracy of the Si:P donor electron ground state could lead to oscillation of the exchange coupling as donor positions are shifted. With our approach to the twoelectron problem, we have calculated the exchange coupling as a function of donor position shift perpendicular to QW plane. Oscillation is observed when gate potential is switched off, and switching-on of gate potential can suppress the exchange oscillation. The exchange coupling as a function of donor position shift along donor coupling axis is also calculated. We find that the exchange oscillation is greatly suppressed at small donor separation and residual oscillation can be observed at large separation. The oscillation behaviors can be explained by analogy to the signal-to-noise ratio.

In conclusion, we have studied in a systematic way the ground state of single $\mathrm{Si}: \mathrm{P}$ donors and the exchange coupling of a coupled donor pair placed in a strained silicon quantum well. Our calculation is of importance in evaluating the prospects and providing practical guidance for a P-donor silicon-based quantum computer.

\section{Acknowledgments}

This work was supported by DARPA DAAD19-01-1-0324 and University of Illinois Research Board.

\section{APPENDIX}

In this appendix we present calculation of gate potential profile for the quantum computer design shown in Fig. 1.

We start from the profile with a single gate electrode in each period. We want to solve the partial differential equation (PDE)

$$
\left[-\nabla^{2}+V(x, z)\right] \phi(x, z)=0
$$

with the following boundary conditions (BC's): 


$$
\begin{gathered}
\phi(x, L)=\phi_{0} \text { for all } x . \\
V(x, z)=\left\{\begin{array}{l}
U_{0} \gg 1 \text { for }|x|<d / 2 \text { and } 0<z<t \\
0 \text { otherwise. }
\end{array}\right. \\
\phi(x,-b)=\phi_{1} \text { for all } x .
\end{gathered}
$$

The system has reflection symmetry about $x=0$ and is periodic in the $x$ direction with period $p$. We divide the system into three regions:

(i) $t<z<L$ (the region above gate electrodes), we have

$$
\begin{aligned}
\phi_{I}(x, z) & =\sum_{n} \cos \left(k_{n} x\right) D_{n}\left[e^{-k_{n}(z-t)}-e^{k_{n}(z+t-2 L)}\right] \\
& +\quad \phi_{0}+D_{0}(z-L)
\end{aligned}
$$

with $k_{n}=2 \pi n / p ; n=0,1,2 \cdots$.

(ii) $0<z<t$ (the region with gate electrodes inside), we have

$$
\phi_{I I}(x, z)=\sum_{m} \beta_{m}(x)\left[B_{m} e^{-q_{m} z}+C_{m} e^{q_{m}(z-t)}\right]
$$

where the basis functions $\beta_{m}(x)$ must satisfy the 1D Schrödinger equation,

$$
\left[-\partial_{x}^{2}+V(x)\right] \beta_{m}(x)=q_{m}^{2} \beta_{m}(x)
$$

Writing $\beta_{m}(x)$ in the form

$$
\beta_{m}(x)=\sum_{n} F_{n m} S_{n} \cos \left(k_{n} x\right) ; S_{n}=\frac{1}{\sqrt{p}}\left[\sqrt{2}\left(1-\delta_{n 0}\right)+\delta_{n 0}\right] .
$$

then $F_{n m}$ can be obtained numerically by solving the eigen-value problem

$$
\sum_{n^{\prime}}\left[k_{n}^{2} \delta_{n n^{\prime}}+V_{n n^{\prime}}\right] F_{n^{\prime} m}=q_{m}^{2} F_{n m}
$$

where

$$
\begin{gathered}
V_{n n^{\prime}}=U_{0} S_{n} S_{n^{\prime}} \int_{-d / 2}^{d / 2} \cos \left(k_{n} x\right) \cos \left(k_{n^{\prime}} x\right) d x= \\
U_{0} S_{n} S_{n^{\prime}}\left[\frac{\sin \left[\left(k_{n^{\prime}}+k_{n}\right) d / 2\right]}{\left(k_{n^{\prime}}+k_{n}\right)}+\frac{\sin \left[\left(k_{n^{\prime}}-k_{n}\right) d / 2\right]}{\left(k_{n^{\prime}}-k_{n}\right)}\right] .
\end{gathered}
$$


(iii) $-b<z<0$ (the region below gate electrodes), we have

$$
\phi_{I I I}(x, z)=\sum_{n} \cos \left(k_{n} x\right) A_{n}\left[e^{k_{n} z}-e^{-k_{n}(z+2 b)}\right]+A_{0}(z+b)+\phi_{1} .
$$

The coefficients are determined by the following BC's:

(i) At $z=t$, we have

$$
\begin{gathered}
S_{n}^{2} \int_{-p / 2}^{p / 2} \cos \left(k_{n} x\right) \phi_{I I}(x, t) d x \\
=S_{n}^{2} \int_{-p / 2}^{p / 2} \cos \left(k_{n} x\right) \phi_{I}(x, t) d x \\
\Rightarrow S_{n} \sum_{m} F_{n m}\left[B_{m} e^{-q_{m} t}+C_{m}\right]=D_{n}\left(1-e^{-2 k_{n}(L-t)}\right) \\
+\delta_{n 0}\left[\phi_{0}+D_{0}(t-L)\right] .
\end{gathered}
$$

Use $\epsilon_{I} \mathbf{E}_{I} \cdot \hat{z}=\epsilon_{I I} \mathbf{E}_{I I} \cdot \hat{z}$, we have

$$
\begin{aligned}
& \epsilon_{I I} S_{n}^{2} \int_{-p / 2}^{p / 2} \cos \left(k_{n} x\right) \partial_{z} \phi_{I I}(x, t) d x \\
= & \epsilon_{I} S_{n}^{2} \int_{-p / 2}^{p / 2} \cos \left(k_{n} x\right) \partial_{z} \phi_{I}(x, t) d x \\
\Rightarrow & \eta S_{n} \sum_{m} F_{n m} q_{m}\left[-B_{m} e^{-q_{m} t}+C_{m}\right] \\
= & -D_{n}\left(1+e^{-2 k_{n}(L-t)}\right) k_{n}+D_{0} \delta_{n 0} .
\end{aligned}
$$

where $\eta \equiv \epsilon_{I I} / \epsilon_{I}$. Eliminate $D_{n}$ (including $D_{0}$ ) by combining the above two equations yields

$$
\begin{gathered}
S_{n} \sum_{m} F_{n m}\left\{\left[1+e^{-2 k_{n}(L-t)}\right]\left[B_{m} e^{-q_{m} t}+C_{m}\right]\right. \\
\left.+q_{m}\left[\left(1-e^{-2 k_{n}(L-t)}\right) \eta / k_{n}\right]\left[-B_{m} e^{-q_{m} t}+C_{m}\right]\right\} \\
=2 \phi_{0} \delta_{n, 0} .
\end{gathered}
$$

where it is understood that $\left[\left(1-e^{-2 k_{n}(L-t)}\right) \eta / k_{n}\right]$ is replaced by $2 \eta(L-t)$ for $n=0$.

(ii) At $z=0$, we have

$$
\begin{aligned}
& S_{n}^{2} \int_{-p / 2}^{p / 2} \cos \left(k_{n} x\right) \phi_{I I}(x, 0) d x \\
= & S_{n}^{2} \int_{-p / 2}^{p / 2} \cos \left(k_{n} x\right) \phi_{I I I}(x, 0) d x
\end{aligned}
$$




$$
\begin{gathered}
\Rightarrow S_{n} \sum_{m} F_{n m}\left[B_{m}+C_{m} e^{-q_{m} t}\right] \\
=A_{n}\left(1-e^{-2 k_{n} b}\right)+\delta_{n 0}\left[A_{0} b+\phi_{1}\right] . \\
\epsilon_{I I} S_{n}^{2} \int_{-p / 2}^{p / 2} \cos \left(k_{n} x\right) \partial_{z} \phi_{I I}(x, 0) d x \\
=\epsilon_{I I I} S_{n}^{2} \int_{-p / 2}^{p / 2} \cos \left(k_{n} x\right) \partial_{z} \phi_{I I I}(x, 0) d x \\
\Rightarrow \zeta S_{n} \sum_{m} F_{n m} q_{m}\left[-B_{m}+C_{m} e^{-q_{m} t}\right] \\
=A_{n}\left(1+e^{-2 k_{n} b}\right) k_{n}+A_{0} \delta_{n 0} .
\end{gathered}
$$

where $\zeta \equiv \epsilon_{I I} / \epsilon_{I I I}$. Eliminate $A_{n}$ (including $A_{0}$ ) by combining the above two equations yields

$$
\begin{gathered}
S_{n} \sum_{m} F_{n m}\left\{\left(1+e^{-2 k_{n} b}\right)\left[B_{m}+C_{m} e^{-q_{m} t}\right]\right. \\
\left.-q_{m}\left[\left(1-e^{-2 k_{n} b}\right) \zeta / k_{n}\right]\left[-B_{m}+C_{m} e^{-q_{m} t}\right]\right\}=2 \phi_{1} \delta_{n, 0} .
\end{gathered}
$$

where it is understood that $\left[\left(1-e^{-2 k_{n} b}\right) \zeta / k_{n}\right]$ is replaced by $2 \zeta b$ for $n=0$. Eqs. (A12) and (A15) constitute a set of coupled linear equations for the coefficients $B_{m}$ and $C_{m}$, which can be solved numerically.

Now we double the period of the system $(-2 p<x<2 p)$ and raise the voltage on the strip with $|x|<d / 2$ (within the first period) by $V_{c}$ and the strip with $2 p-d / 2<|x|<2 p$ by $V_{g}$, and similarly for all other periods. The solution to $\phi(x, z)$ in regions I and III are written in the same form as the case with $V_{g}=0$, except that $k_{n}^{\prime} s$ are replaced by $k_{n}^{\prime} \pi n / 2 p ; n=0,1,2 \cdots$ and the unknown coefficients are denoted $A_{n}^{\prime}$ and $D_{n}^{\prime}$. In region II, we need a set of basis functions

$$
\beta_{m}^{\prime}(x)=\sum_{n} F_{n m}^{\prime} S_{n}^{\prime} \cos \left(k_{n}^{\prime} x\right) ; S_{n}^{\prime}=\frac{1}{2 \sqrt{p}}\left[\sqrt{2}\left(1-\delta_{n 0}\right)+\delta_{n 0}\right] .
$$

where $F_{n m}^{\prime}$ are solved similarly as in the $V_{g}=0$ case with

$$
\begin{aligned}
V_{n n^{\prime}}= & U_{0} S_{n}^{\prime} S_{n^{\prime}}^{\prime} 2\left[\int_{0}^{d / 2} \cos \left(k_{n}^{\prime} x\right) \cos \left(k_{n^{\prime}}^{\prime} x\right)\right. \\
& +\int_{p-d / 2}^{p+d / 2} \cos \left(k_{n}^{\prime} x\right) \cos \left(k_{n^{\prime}}^{\prime} x\right)
\end{aligned}
$$




$$
\begin{gathered}
\left.+\int_{2 p-d / 2}^{2 p} \cos \left(k_{n}^{\prime} x\right) \cos \left(k_{n^{\prime}}^{\prime} x\right)\right] d x \\
=U_{0} \delta_{n, n^{\prime}}+U_{0} S_{n} S_{n^{\prime}}\left\{\frac{\sin \left[\left(k_{n^{\prime}}^{\prime}+k_{n}^{\prime}\right) d / 2\right]}{\left(k_{n^{\prime}}^{\prime}+k_{n}^{\prime}\right)}+\frac{\sin \left[\left(k_{n^{\prime}}^{\prime}-k_{n}^{\prime}\right) d / 2\right]}{\left(k_{n^{\prime}}^{\prime}-k_{n}^{\prime}\right)}\right. \\
-\frac{\sin \left[\left(k_{n^{\prime}}^{\prime}+k_{n}^{\prime}\right)(p-d / 2)\right]}{\left(k_{n^{\prime}}^{\prime}+k_{n}^{\prime}\right)}-\frac{\sin \left[\left(k_{n^{\prime}}^{\prime}-k_{n}^{\prime}\right)(p-d / 2)\right]}{\left(k_{n^{\prime}}^{\prime}-k_{n}^{\prime}\right)} \\
+\frac{\sin \left[\left(k_{n^{\prime}}^{\prime}+k_{n}^{\prime}\right)(p+d / 2)\right]}{\left(k_{n^{\prime}}^{\prime}+k_{n}^{\prime}\right)}+\frac{\sin \left[\left(k_{n^{\prime}}^{\prime}-k_{n}^{\prime}\right)(p+d / 2)\right]}{\left(k_{n^{\prime}}^{\prime}-k_{n}^{\prime}\right)} \\
\left.-\frac{\sin \left[\left(k_{n^{\prime}}^{\prime}+k_{n}^{\prime}\right)(2 p-d / 2)\right]}{\left(k_{n^{\prime}}^{\prime}+k_{n}^{\prime}\right)}-\frac{\sin \left[\left(k_{n^{\prime}}^{\prime}-k_{n}^{\prime}\right)(2 p-d / 2)\right]}{\left(k_{n^{\prime}}^{\prime}-k_{n}^{\prime}\right)}\right\} .
\end{gathered}
$$

Furthermore, we add a particular solution defined as

$$
\phi_{p}(x)=\left\{\begin{array}{l}
V_{c} \text { for }|x|<d / 2 \\
V_{c} \frac{p-d / 2-|x|}{p-d} \text { for } d / 2<|x|<p-d / 2 \\
0 \text { for } p-d / 2<|x|<p+d / 2 \\
V_{g} \frac{|x|-p-d / 2}{p-d} \text { for } p+d / 2<|x|<2 p-d / 2 \\
V_{g} \text { for } 2 p-d / 2<|x|<2 p .
\end{array}\right.
$$

Note that $\phi_{p}(x)$ satisfies the homogeneous PDE and it leads to correct potential difference between the adjacent metallic strips in region II. Thus, the potential function in region II takes the form

$$
\begin{gathered}
\phi_{I I}(x, z)=\phi_{p}(x)+ \\
\sum_{m} \beta_{m}^{\prime}(x)\left[B_{m}^{\prime} e^{-m \pi z / 2 p}+C_{m}^{\prime} e^{m \pi(z-t) / 2 p}\right] .
\end{gathered}
$$

After matching the boundary conditions, we obtain two sets of coupled equations similar to Eqs. (A12) and (A15), except that we subtract a term $\left[1+e^{-2 k_{n}^{\prime}(L-t)}\right] F_{p n}$ and $\left[1+e^{-2 k_{n}^{\prime} b}\right] F_{p n}$ from the right hand sides of these equations.

$$
\begin{gathered}
F_{p n} \equiv S_{n}^{\prime 2} \int_{-2 p}^{2 p} \cos \left(k_{n}^{\prime} x\right) \phi_{p}(x) d x \\
=2 V_{c} S_{n}^{\prime 2}\left\{\sin \left[k_{n}^{\prime}(p-d / 2)\right]\right. \\
+\frac{d}{2(p-d)}\left[\sin \left[k_{n}^{\prime}(p-d / 2)\right]-\sin \left(k_{n}^{\prime} d / 2\right)\right] \\
\left.-\left.\frac{1}{p-d}\left[x \sin k_{n}^{\prime} x+\cos k_{n}^{\prime} x / k_{n}^{\prime}\right]\right|_{d / 2} ^{p-d / 2}\right\} / k_{n}^{\prime} \\
+V_{g} \delta_{n 0}-2 V_{g} S_{n}^{\prime 2}\left\{\sin \left[k_{n}^{\prime}(p+d / 2)\right]\right.
\end{gathered}
$$




$$
\begin{gathered}
+\frac{2 p-d / 2}{(p-d)}\left[\sin \left[k_{n}^{\prime}(2 p-d / 2)\right]-\sin \left(k_{n}^{\prime}(p+d / 2)\right]\right. \\
\left.-\left.\frac{1}{p-d}\left[x \sin k_{n}^{\prime} x+\cos k_{n}^{\prime} x / k_{n}^{\prime}\right]\right|_{p+d / 2} ^{2 p-d / 2}\right\} / k_{n}^{\prime} .
\end{gathered}
$$

The region $-a<z<0$ is now filled with a dielectric $\left(\mathrm{Si}_{3} \mathrm{~N}_{4}\right)$ with dielectric constant $\epsilon_{a}$ (the dielectric screening constants for the silicon layer and SiGe layer are close to each other, so we ignore their differences and use $\epsilon \simeq 12$ ). In this region, the potential function is replaced by

$$
\begin{gathered}
\phi_{a}(x, z)=\sum_{n} \cos \left(k_{n}^{\prime} x\right)\left[\bar{A}_{n} e^{k_{n}^{\prime} z}-R_{n} e^{-k_{n}^{\prime}(z+2 a)}\right] \\
+\bar{A}_{0}(z+a)+\phi_{a}
\end{gathered}
$$

Matching $\phi_{a}(x, z)$ with $\phi_{I I I}(x, z)$ at boundary $z=-a$ gives

$$
\begin{aligned}
& \bar{A}_{n}=[\xi(1+\gamma)+(1-\gamma)] A_{n}^{\prime} / 2 \equiv \chi^{+} A_{n}^{\prime} \\
& R_{n}=[\xi(1+\gamma)-(1-\gamma)] A_{n}^{\prime} / 2 \equiv \chi^{-} A_{n}^{\prime}
\end{aligned}
$$

and

$$
\phi_{a}=A_{0}^{\prime}(b-a)+\phi_{1}, \bar{A}_{0}=\xi A_{0}^{\prime}
$$

where $\gamma=e^{-2 k_{n}^{\prime}(b-a)}$ and $\xi=\epsilon_{I I I} / \epsilon_{a}$. Matching $\phi_{a}(x, z)$ with $\phi_{I I}(x, z)$ at boundary $z=0$ gives

$$
\begin{gathered}
S_{n}^{\prime} \sum_{m} F_{n m}^{\prime}\left\{\left(\chi^{+}+\chi^{-} e^{-2 k_{n}^{\prime} a}\right)\left[B_{m}^{\prime}+C_{m}^{\prime} e^{-q_{m}^{\prime} t}\right]\right. \\
\left.-q_{m}^{\prime}\left[\left(\chi^{+}-\chi^{-} e^{-2 k_{n}^{\prime} a}\right) \zeta / k_{n}^{\prime}\right]\left[-B_{m}^{\prime}+C_{m}^{\prime} e^{-q_{m}^{\prime} t}\right]\right\} \\
=2 \phi_{a} \delta_{n, 0}-\left[\chi^{+}+\chi^{-} e^{-2 k_{n}^{\prime} a}\right] F_{p n} .
\end{gathered}
$$

where $\zeta=\epsilon_{I I} / \epsilon_{a}$. It is understood that $\left(\chi^{+}-\chi^{-} e^{-2 k_{n}^{\prime} a}\right) \zeta / k_{n}^{\prime}$ will be replaced by $2 \zeta[b+(\xi-1) a]$ for $n=0$.

[1] M. A. Nielsen, I. L. Chuang, Quantum Computation and Quantum Information (Cambridge University Press, 2000).

[2] B. E. Kane, Nature(London) 393133 (1998). 
[3] R. Vrijen, E. Yablonovitch, K. Wang, H. W. Jiang, A. Balandin, V. Roychowdhury, Tal Mor and D. DiVincenzo, Phys. Rev. A 62012306 (2000).

[4] M. Friesen, P. Rugheimer, D. E. Savage, M. G. Lagally, D. W. van der Weide, R. Joynt, and M. A. Eriksson, Phys. Rev. B 67, 121301(R) (2003).

[5] A. J. Skinner, M. E. Davenport, and B. E. Kane, Phys. Rev. Lett. 90087901 (2003).

[6] C. D. Hill, L. C. L. Hollenberg, A. G. Fowler, C. J., Wellard, A. D. Greentree, and H. -S. Goan, arXiv: quant-ph/0411104

[7] J. R. Tucker and T. -C. Shen, Quant. Info. Proc., 3, 105 (2004).

[8] D. P. Divencenzo, D. Bacon, J. Kempe, G. Bukard, and K. B. Whaley, Nature, 408, 339 (2000).

[9] B. E. Kane, N. S. McAlpine, A. S. Dzurak, R. G. Clark, G. J. Milburn, H. B. Sun, and H. Wiseman, Phys. Rev. B 61, 2961 (2000).

[10] C. Tahan, M. Friesen, and R. Joynt, Phys. Rev. B 66, 035314 (2002).

[11] A. M. Tyryshkin, S. A. Lyon, A. V. Astashkin, and A. M. Raitsimring, Phys. Rev. B 68, 193207 (2003).

[12] R. de Sousa and S. Das Sarma, Phys. Rev. B 67, 033301 (2003); R. de Sousa and S. Das Sarma, Phys. Rev. B 68, 115322(2003).

[13] E. Abe, K. M. Itoh, J. Isoya, and S. Yamasaki, Phys. Rev. B 70, 033204 (2004).

[14] C. Tahan and R. Joynt, Phys. Rev. B 71, 075315 (2005).

[15] G. Feher and E.A. Gere, Phys. Rev. 114, 1245 (1959).

[16] G. Smit, S. Rogge, J. Caro, and T. Klapwijk, Phys. Rev. B 68, 193302 (2003).

[17] M. Friesen, arXiv: cond-mat/0501267

[18] G. D. J. Smit, S. Rogge, J. Caro, and T. M. Klapwijk, Phys. Rev. B 70, 035206 (2004).

[19] B. Koiller, X. Hu, and S. Das Sarma, Phys. Rev. Lett. 88, 027903 (2002).

[20] A. Fang, Y. C. Chang and J. R. Tucker, Phys. Rev. B 66155331 (2002).

[21] B. Koiller, X. Hu, and S. Das Sarma, Phys. Rev. B 66, 115201 (2002).

[22] C. J. Wellard, L. C. L. Hollenberg, F. Parisoli, L. Kettle, H.-S. Goan, J. A. L. McIntosh, and D. N. Jamieson, Phys. Rev. B 68, 195209 (2003).

[23] B. Koiller,R. B. Capaz, X. Hu, and S. Das Sarma, Phys. Rev. B 70, 115207 (2004).

[24] C. J. Wellard, L. C. L. Hollenberg, L. Kettle, and H.-S. Goan, arXiv: cond-mat/0402642.

[25] P. Y. Yu and M. Cardona, Fundamentals of Semiconductors, 3rd edition(Springer-Verlag, 2003). 
[26] W. Kohn and J. M. Luttinger, Phys. Rev. 97, 1721 (1955); W. Kohn and J. M. Luttinger, Phys. Rev. 98, 915 (1955).

[27] S. T. Pantelides, Rev. Mod. Phys. 50, 797 (1978).

[28] A. K. Ramdas and S. Rodriguez, Rep. Prog. Phys. 44, 1297 (1981).

[29] R. A. Faulkner, Phys. Rev. 184, 713 (1969).

[30] S. T. Pantelides and C. T. Sah, Phys. Rev. B 10, 621 (1974).

[31] H. Fritzsche, Phys. Rev. 125, 1560 (1962).

[32] R. Resta, Phys. Rev. B 16, 2717 (1977).

[33] A. Blom, M. A. Odnoblyudov, I. N. Yassievich and K. -A. Chao, Phys. Rev. B 68, 165338 (2003).

[34] A. S. Martins, R. B. Capaz, and Belita Koiller, Phys. Rev. B 69, 085320 (2004).

[35] T. B. Boykin, G. Klimeck, M. A. Eriksson, M. Friesen, S. N. Coppersmith, P. von Allmen, F. Oyafuso, and S. Lee, Appl. Phys. Lett. 84, 115 (2004).

[36] T. B. Boykin, G. Klimeck, M. Friesen, S. N. Coppersmith, P. von Allmen, F. Oyafuso, and S. Lee, Phys. Rev. B 70, 165325 (2004).

[37] S. Huzinaga, J. Chem. Phys. 42, 1293 (1965).

[38] J. L. O’Brien, S. R. Schofield, M. Y. Simmons, R. G. Clark, A. S. Dzurak, N. J. Curson, B. E. Kane, N. S. McAlpine, M. E. Hawley and G. W. Brown, Phys. Rev. B 64, 161401(R) (2001).

[39] Herring and M. Flicker Phys. Rev. 134, A362 (1964).

[40] M. Friesen, R. Joynt and M. A. Eriksson, Appl. Phys. Lett. 81, 4619 (2002).

[41] J. Schliemann, D. Loss and A. McDonald, Phys. Rev. B 63085311 (2001).

[42] M. Grace, C. Brif, H. Rabitz, I. Walmsley, R. Kosut and D. Lidar, arXiv:quant-ph/0412059.

[43] S. N. Coppersmith, S. Lee and P. von Allmen, arXiv: cond-mat/0408542.

[44] S. R. Schofield, N. J. Curson, M. Y. Simmons, F. J. Rue $\beta$, T. Hallam, L. Oberbeck, and R. G. Clark, Phys. Rev. Lett. 91, 136104 (2003). 
FIG. 1: The composite 3-spin "universal exchange" qubit of Si:P donors in a Kane-type architechture with the integrated SET readout. From bottom to top, the heterostructure cross section consists of a thick, n-doped ground layer, a $28 \mathrm{~nm}$ undoped $S i_{0.7} G e_{0.3}$ tunnel barrier, a 10-nm Si quantum well(with phosphorous donor array embedded in the middle), a $7 \mathrm{~nm}$ undoped $S i_{0.7} G e_{0.3}$ tunnel barrier, a $5 \mathrm{~nm} \mathrm{Si} i_{3} N_{4}$ layer and lithographically-patterned metallic top gates. The logic qubit is represented by the 2-dimensional subspace $\left|\mathbf{S}, \mathbf{S}_{\mathbf{z}}\right\rangle=\left|\frac{1}{2}, \frac{1}{2}\right\rangle$ of the three neighboring donor spins, with logic zero $\left|0_{L}\right\rangle=|S\rangle|\uparrow\rangle$ and logic one $\left|1_{L}\right\rangle=\sqrt{2 / 3}\left|T_{+}\right\rangle|\downarrow\rangle-\sqrt{1 / 3}\left|T_{0}\right\rangle|\uparrow\rangle$.

FIG. 2: The energies of two lowest states of a single Si:P donor and their splitting as a function of quantum well width. For convenience, the valley splitting is plotted in negative values.

FIG. 4: The valley splitting of (a) Si:P donor ground state and (b) lowest quantum well state, as a function of quantum well width. The dotted lines connect points with a spacing of $a_{0} / 2$. The inset of (a) shows the valley splitting of Si:P ground state as a function of donor position shift from the quantum well central plane, for a QW width fixed at 9nm.

FIG. 5: The gate potential(measured in units of $R y^{*}$ ) profile at different gate voltages: (a) $V_{c}=$ $-2.1 \mathrm{~V}$; (b) $V_{c}=-0.8 \mathrm{~V}$; (c) $V_{c}=-0.6 \mathrm{~V}$ and $(\mathrm{d}) V_{c}=-3.2 \mathrm{~V} . V_{g}=-1.8$ for all these four cases. In each figure, the potential shape is plotted along $\mathrm{x}$ axis, with three different distances $(15,17,19 \mathrm{~nm})$ from the top gates. The doping plane in our QC device is $17 \mathrm{~nm}$ below the top gates.

FIG. 6: The exchange coupling of a donor pair as a function of quantum well width. The donor separation is fixed at $10 a_{B}{ }^{*}$. The inset shows the change of exchange coupling as the QW width is varied in atomic scale, where the calculating points are spaced by $a_{0} / 4 \simeq 1.36 \AA$.

FIG. 3: The comparison of the averaged 1D charge distribution of Si:P ground state in a quantum well with width $6 \mathrm{~nm}$ and 10nm. (a) and (b) shows the charge distribution along $\mathrm{z}$ and $\mathrm{x}$ axis, respectively. The inset of (b) shows the charge distribution along y axis. 
FIG. 7: (a) The exchange coupling of a donor pair separated by $10 a_{B}{ }^{*}$ as a function of central gate voltages. The three curves correspond to three different values of side gate voltages $V_{g}=-1.4,-1.8$ and $-2.0 \mathrm{~V}$, respectively. The isolated point (filled square) on the $V_{c}=0$ axis corresponds to the exchange coupling without any gate potential $\left(V_{c}=V_{g}=0\right)$. (b) The dependence of exchange coupling on central gate voltages, with $V_{g}$ fixed at $-1.8 \mathrm{~V}$. The three curves correspond to three different donor separations $R=8,10$, and $12 a_{B}{ }^{*}$, respectively. Both (a) and (b) are plotted in logarithmic scale for the exchange coupling. The quantum well width is fixed at $10 \mathrm{~nm}$.

FIG. 8: The averaged 1D two-electron charge distribution of singlet (solid) and triplet (dotted) states along the inter-donor axis, for different gate voltages. The side gate voltage is $V_{g}=-1.8 \mathrm{~V}$ for (b), (c) and (d). The charge distributions for singlet and triplet almost coincide for weakly coupled donor pairs, as shown in (a) and (d). The gate-off exchange coupling is very close to the gate-on case with $V_{c}=-2.1 \mathrm{~V}$ and $V_{g}=-1.8 \mathrm{~V}$, while their charge distributions are apparently different, as shown in (a). It is clearly demonstrated that the gate voltages can tune the overlap between neighboring donor electron wave functions and consequently, the exchange coupling.

FIG. 9: The exchange coupling as a function of donor position shift away from the quantum well central plane: (a)gate off; (b) gate on $\left(V_{c}=-1.6 \mathrm{~V}\right.$ and $\left.V_{g}=-1.8 \mathrm{~V}\right)$. The donor pair remains on the [100] axis, with their separation fixed at $10 a_{B}{ }^{*}$. The inset of (a) plots the overlap of single-particle wave functions(for singlet state) as a function of donor position shift, which shows the same pattern as the exchange coupling. The dotted line in (b) is an exponential fitting curve, illustrating the significant effect of gate potential on the exchange coupling for donors at different distances from top gates.

FIG. 10: The exchange coupling(plotted in logarithmic scale) as a function of donor separation. The donor pair remains on the [100] axis, with their separation varied from 70 to 90, in units of half lattice constant, $a_{0} / 2$. The dotted line is an exponential fitting curve, appearing as a straight line in the logarithmic-scale plotting . 


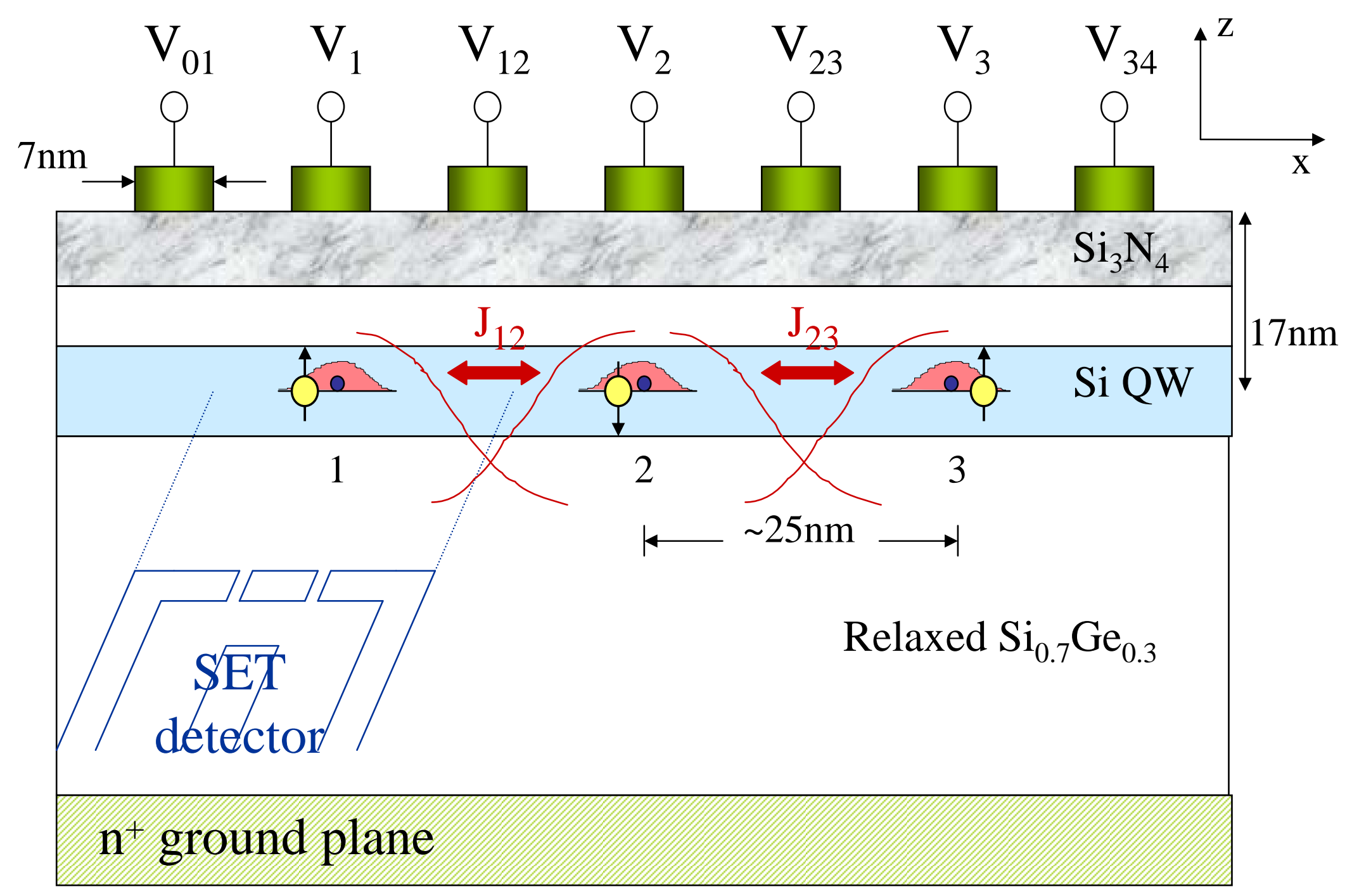




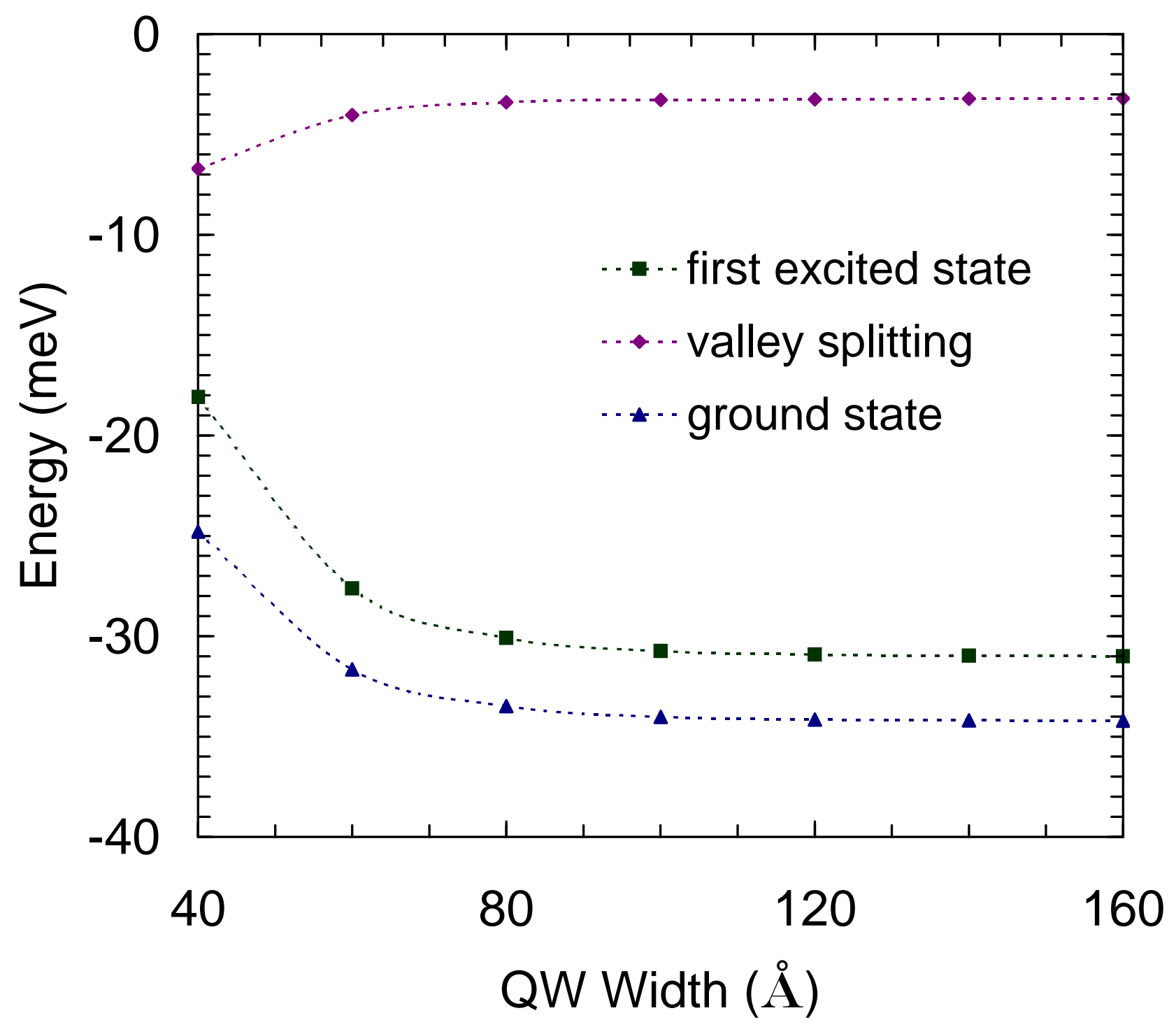



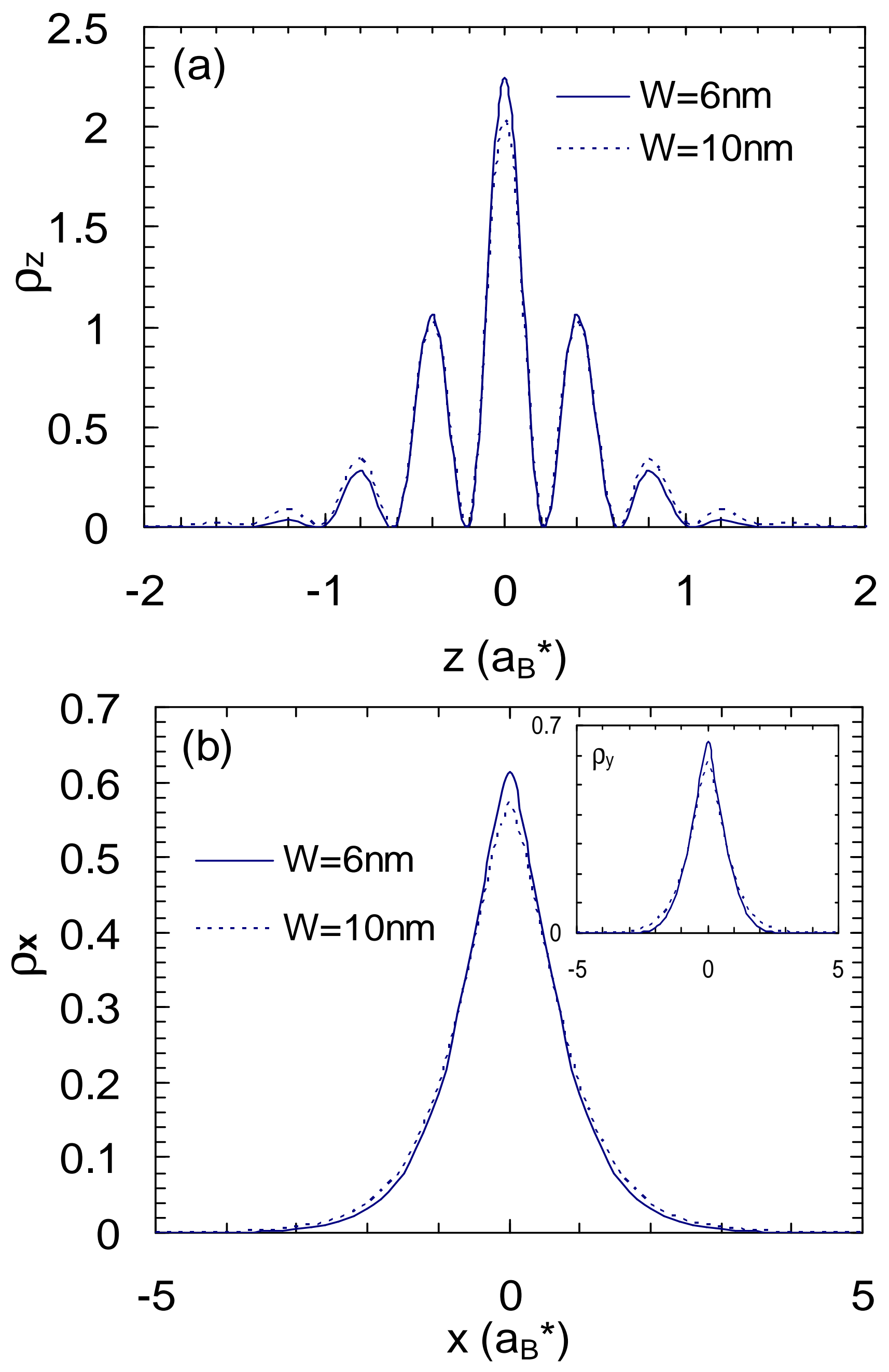

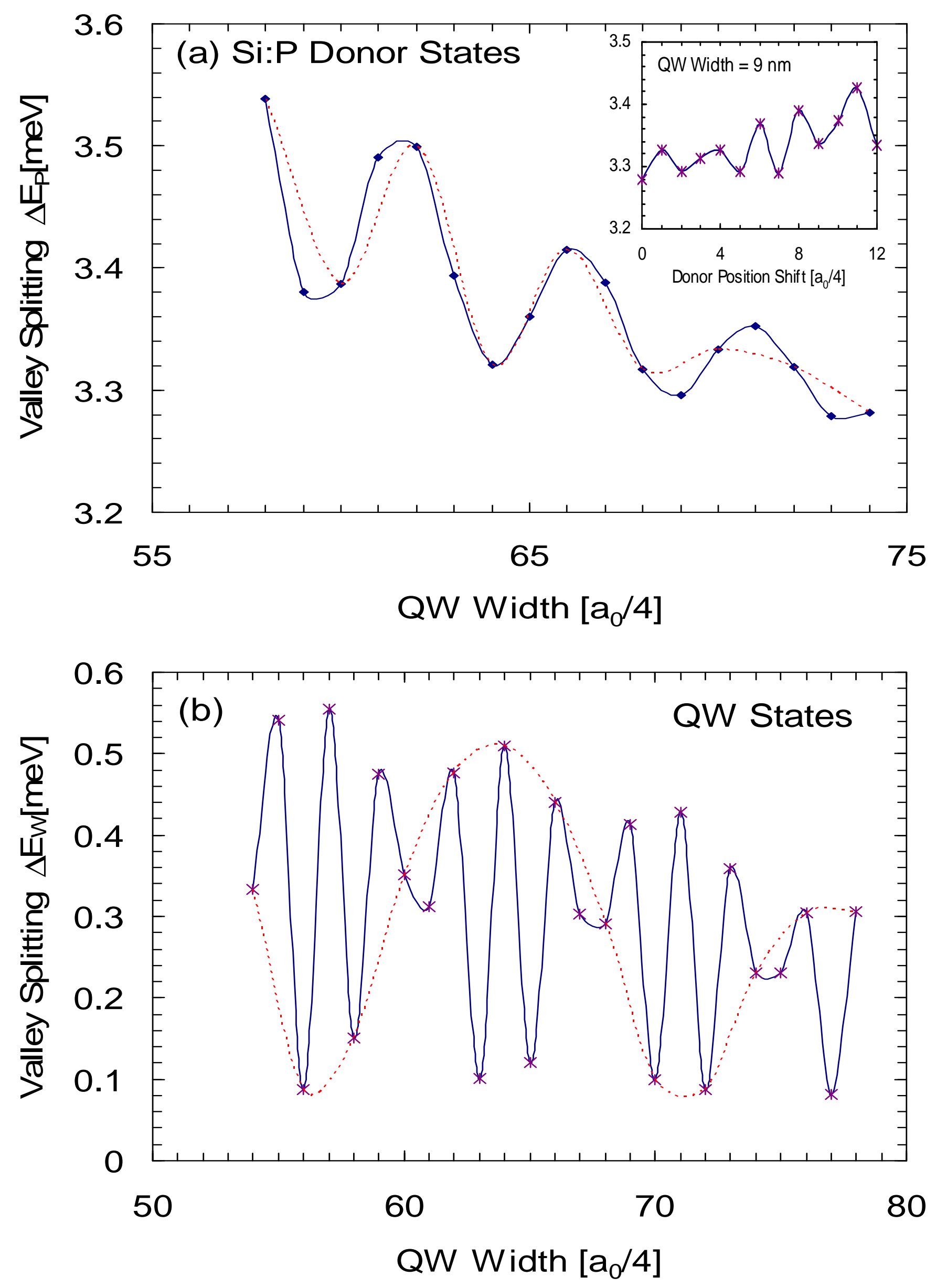

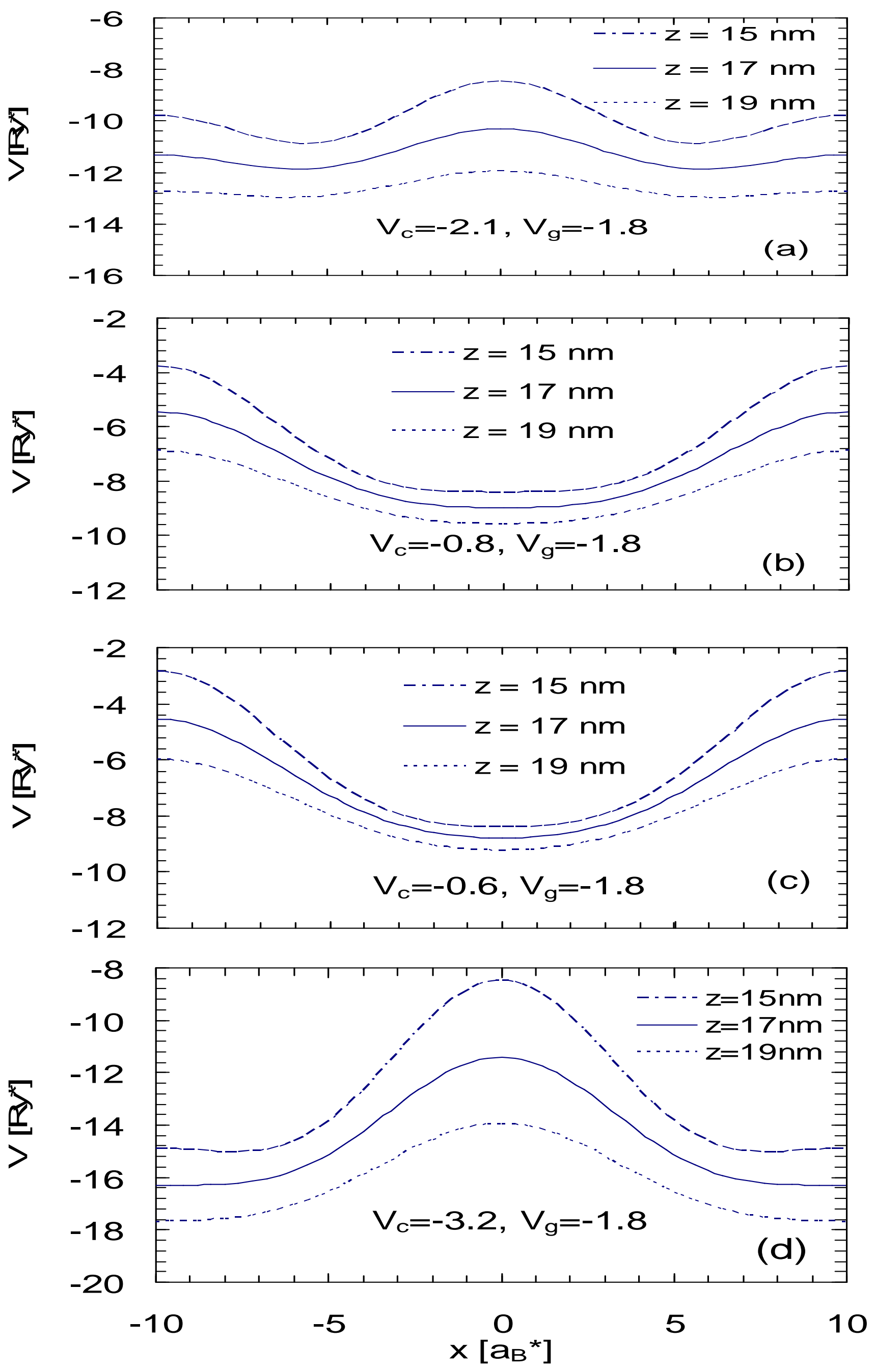


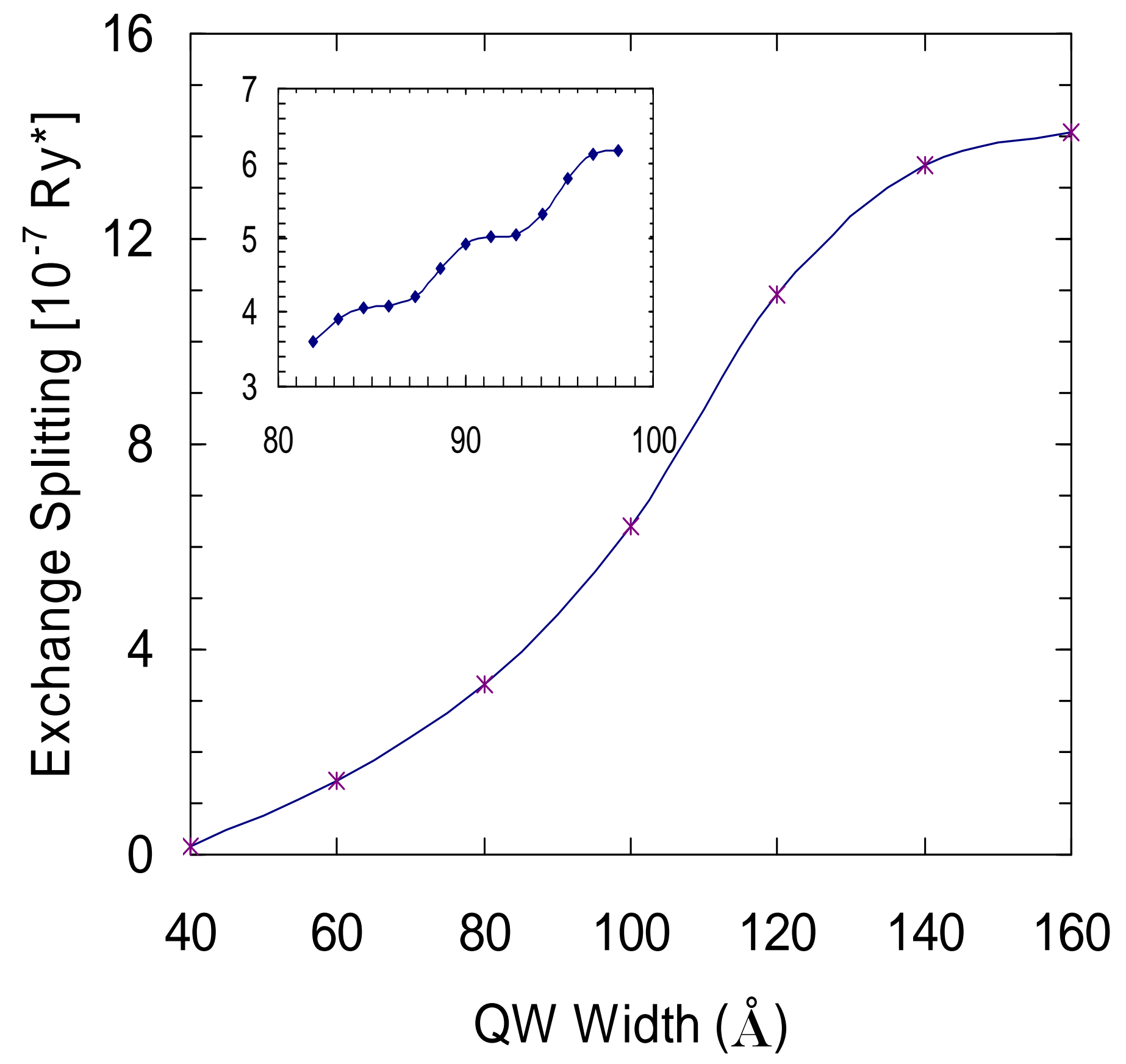




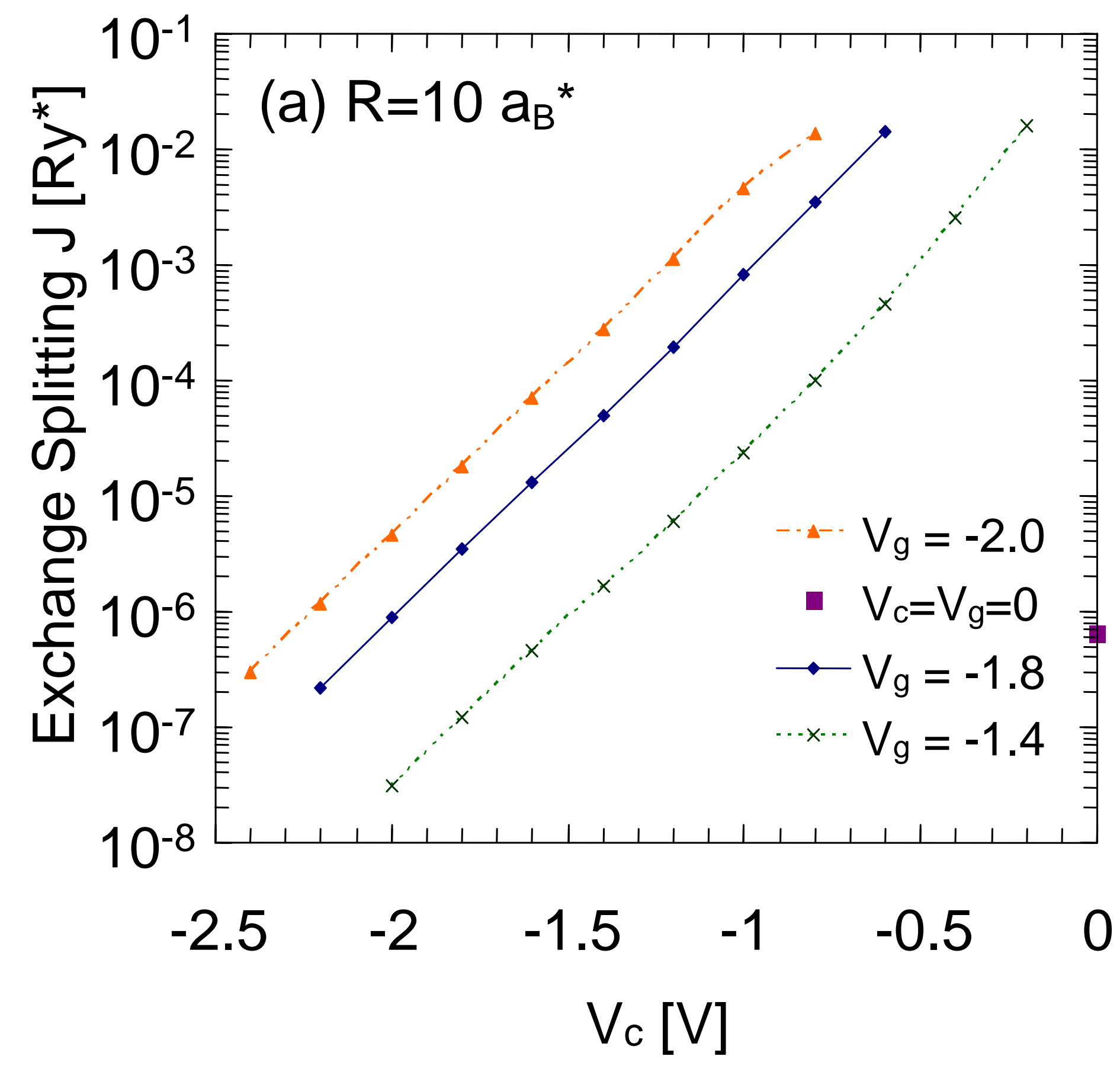




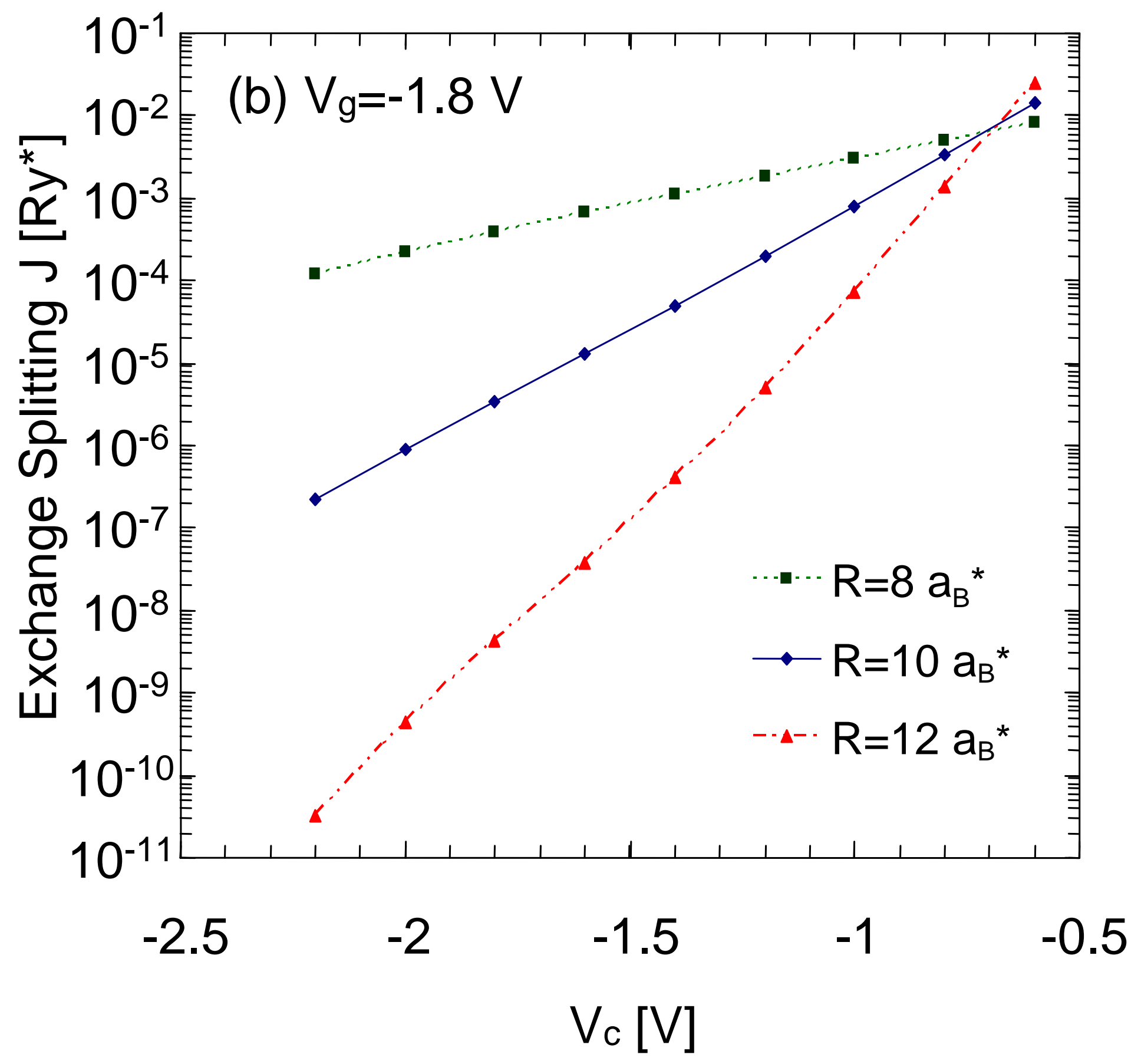



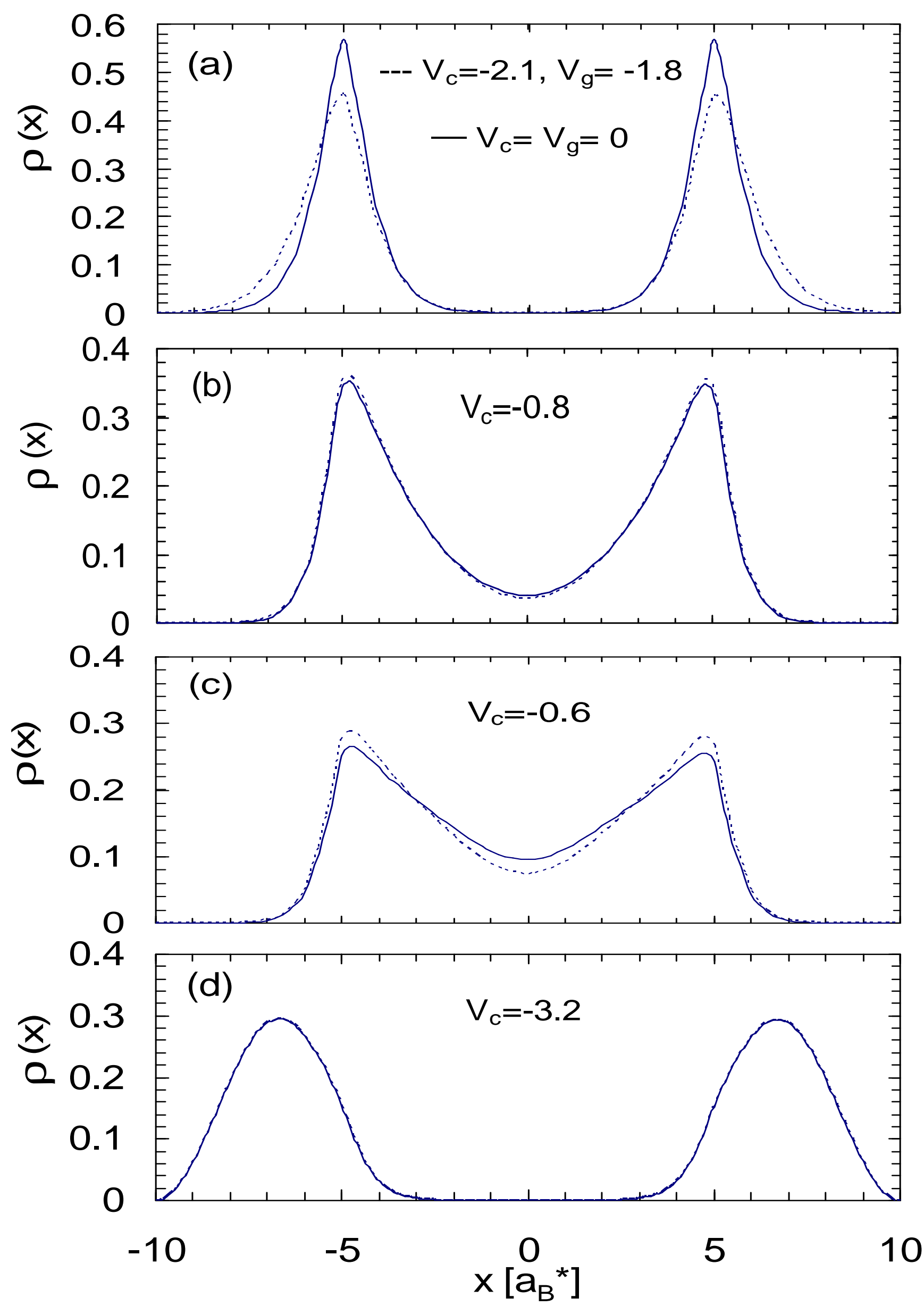

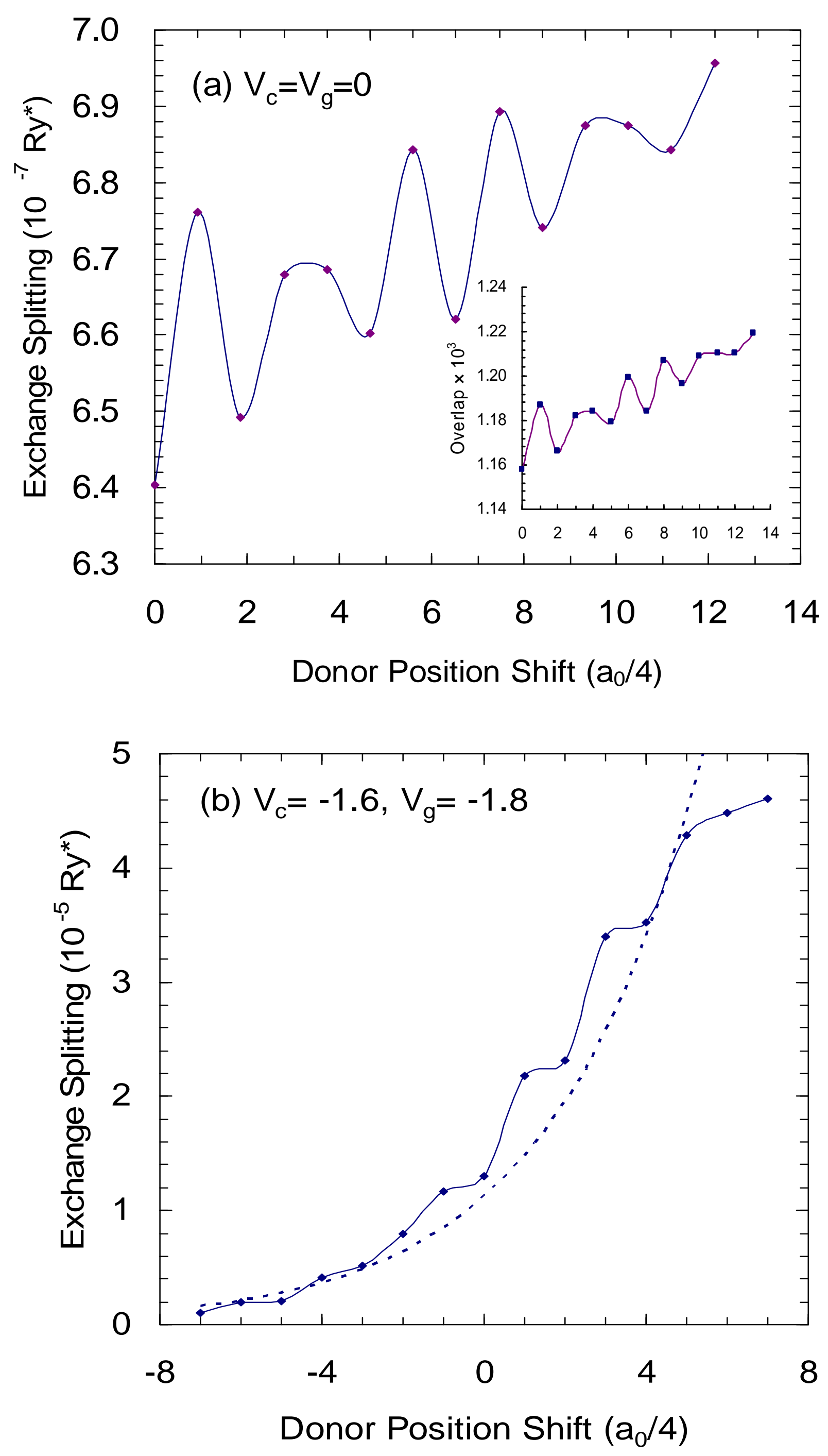


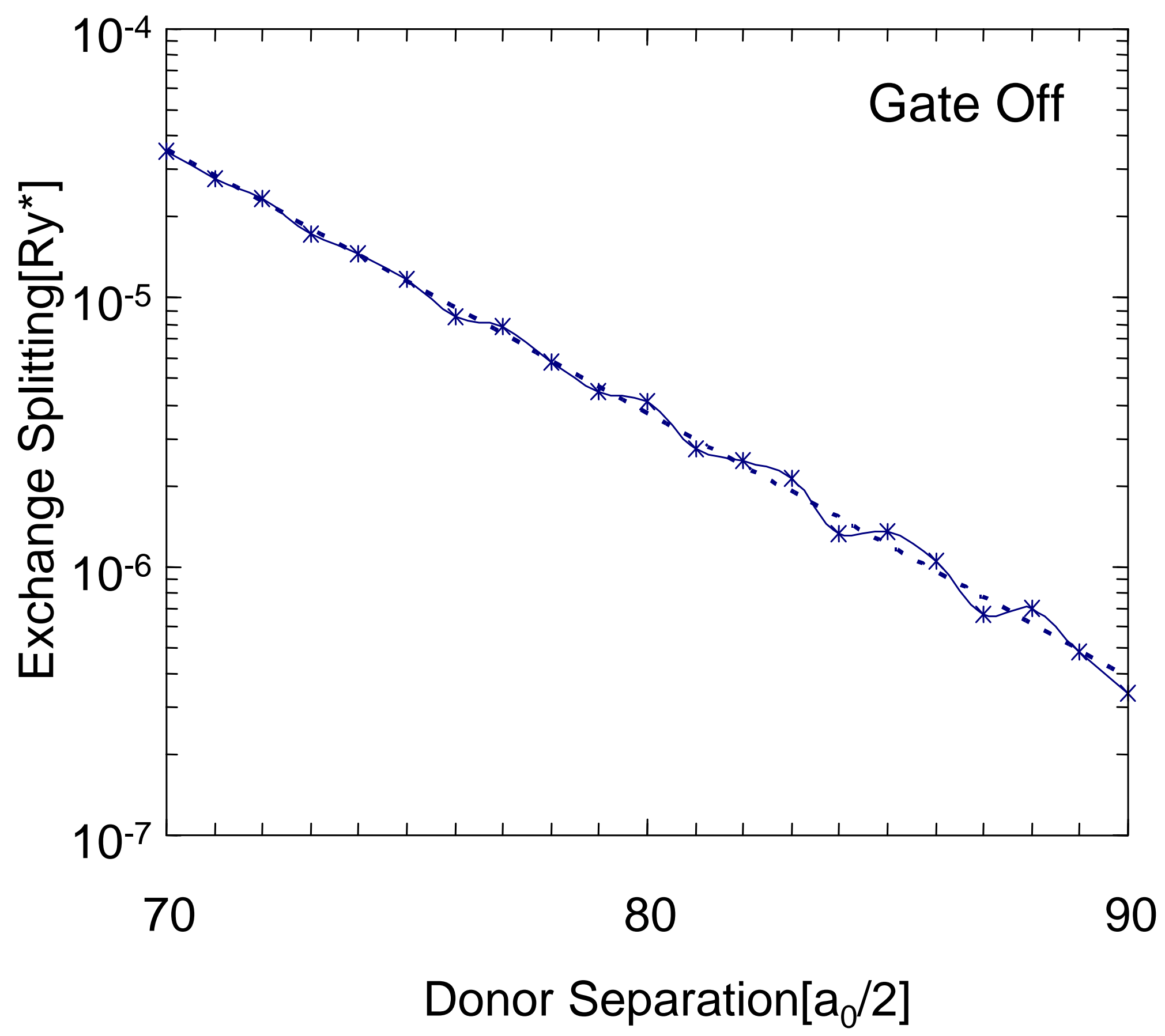

